\title{
27. VARIABLE STARS (ETOILES VARIABLES)
}

PRESIDENT: M. BREGER

VICE PRESIDENT: J.R. Percy

ORGANIZING COMMITTEE: T. G. Barnes. J. Christensen-Dalsgaard, R. E. Gershberg. M. Jerzykiewicz, L. N. Mavridis, M. Rodono, M. A. Smith. B. Szeidl, A. M. van Genderen, B. Warner

\section{Introduction}

Conm. 27 sponsors three special services for the astronomical community: the Information Bulletin On :ariable Stars (IBVS). the Archives of Unpublished Observations of Variable Stars, and the General Catalog of Variable Stars. The news from the centers organizing these activities can be found in Section 2. The subsequent sections present a summary of the developments between 1987 and 1990 in the various scientific fields written by selected experts. We are grateful to the authors for their excellent reviews.

The commission expresses its thanks to Bela Szeidl of Konkoly Observatory for having edited the IBVS for more than two decades. This important and time-consuming task will be taken over by L. Szabados and K. Oláh, also at Konkoly Observatory.

\section{Commission Activities}

\section{A. INFORMATION BULLETIN ON VARIABLE STARS (L. Szabados)}

During the period covered by this report, the number of issues of the IBVS progressed from No. 3042 (July. 1987) to 3487 (June, 1990). The percentage of camera-ready manuscripts is increasing. which helps to decrease the work connected with publication. For economic reasons the issues of IBVS are usually mailed once a month. In the most unfortunate case accepted manuscripts can take six weeks for publication.

Regrettably, several recently submitted manuscripts have been lost in the mail. Consequently, the manuscripts received are now acknowledged (allow about three to four weeks for the mail services). Short notes containing only alphanumeric characters can be submitted via $E$-mail (see the address below).

Potential contributors to the IBVS can request instructions concerning the submission of manuscripts. The address of all correspondence is as follows:

The Editors, IBVS

Konkoly Observatory

P. O. Box 67

$\mathrm{H}-1525$ Budapest

Hungary

\section{E-Mail: H1036IBV@ELLA.UUCP.}

The circulation of IBVS now exceeds 600. However, this number cannot be significantly increased in the future. The mailing list of the IBVS can only be extended to newly established professional observatories and university departments. Membership in Commission 27 does not include receiving a personal copy of the IBVS. The editors of the IBVS express their gratitude to those colleagues who have sent some exchange material (journals, books, conference proceedings, etc.) in return for the IBVS.

After two decades of editing the IBVS. Bela Szeidl will retire from the IBVS starting with issue 3500. The new co-editor will be Dr. Katalin Olảh.

\section{B. ARCHIVES OF UNPUBLISHED OBSERVATIONS (Michel Breger)}

The Archives of Unpublished Observations of Variable Stars was created to provide permanent archives in different parts of the world. The Archives can replace lengthy and expensive tables in scientific publications by a single reference to the archival file number. Furthermore, many valuable observations are never used for scientific publications, and the Archives makes such observations available to other astronomers at a time when they might become very important.

The major addition to the Archives has been the possibility of utilizing electronic means for the transmission of data. such as electronic mail. diskette or magnetic tape. However. the user is urged to compare the electronically submitted data with a paper copy to guard against unexpected losses or 
gains of information. Older files, which were originally submitted on paper only, are not yet available in computer readable form.

The r: umber of completed files with data sets has grown to 211 , and considerably more files have been assigned to data not yet received. Rapid retrieval of past files at no cost has been reported from the depositories.

Detailed reports on the contents of recent as well as older files can be found in PASP 100, 751 , 1988 and Bull. Centre Donn. Stell, 35. 93. 1988, while a description of how to use the Archives was given in IBVS 3422, 1990 as well as Bull. Centre Donn. Stell. 37, 147, 1990 and IAPP Comm. 40, 36, 1990.

Astronomers who wish to obtain copies of specific unpublished observations of variable stars may do so by requesting whole files by file number from one of the three archives listed below. Should eletronic data transmission be desired for those files available in computer readable form, only the Centre de Donnees Stellaires should be contacted. The addresses are:

\section{Dr. P. Dubois}

Centre de Données Stellaires

11. Rue de |'Université

F-67000 Strasbourg

France

\author{
P. D. Hingley, Librarian \\ Royal Astronomical Society \\ Burlington House \\ London. W1V ONL \\ Great Britain
}

Dr. Yu. S. Romanov

Astronomical Observatory

Shevchenko Park

Odessa 270014

U.S.S.R.

Astronomers, who wish to submit new files, should obtain a file number by contacting the coordinator at

Prof. Dr. Michel Breger

Institut für Astronomie

Türkenschanzstraße 17

A-1180 Wien

Austria.

Electronic transmission of data should be accompanied by a single paper copy of both the cover sheet and the data and submitted together with information on the previously assigned file number to P. Dubois in Strasbourg. If electronic transmission of data is not used, three paper copies of the data and five copies of the cover sheet should be submitted to M. Breger. completed:

Since our previous report to IAU Commission 27, the following files have been assigned and

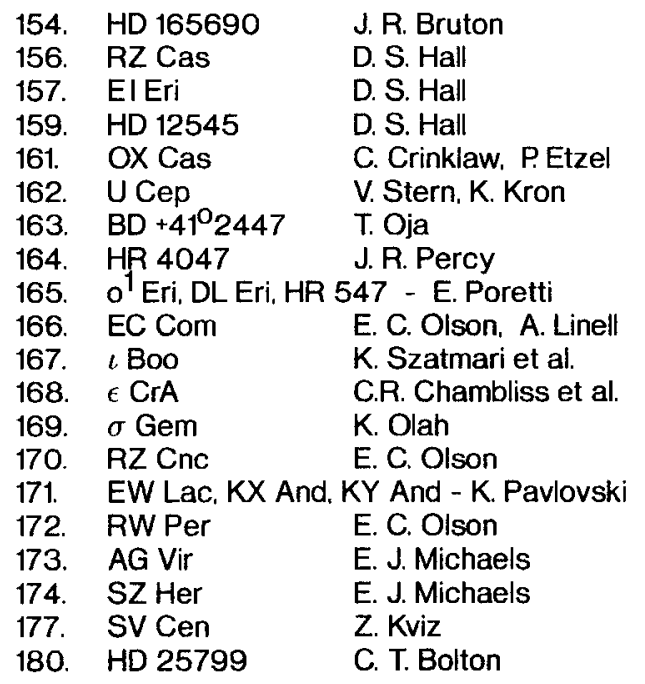

\begin{tabular}{|c|c|c|}
\hline Do. & $\epsilon$ Per & J. R. Percy \\
\hline 02. & ST Ind & K. Walter. H. Duerbeck \\
\hline 55. & $\alpha$ Lup & J. Cuypers \\
\hline 06. & $\beta$ Cru & J. Cuypers \\
\hline j & $\delta$ Ceti & J. Cuypers \\
\hline 08. & $\nu$ Eri & J. Cuypers \\
\hline & $\sigma \mathrm{Sco}$ & J. Cuypers \\
\hline & $\tau^{1}$ Lup & J. Cuypers \\
\hline & KU Cyg & E. C. Olson \\
\hline 2 & 8 apsidal motion sy & stems - D. B. Caton \\
\hline 15 & $4 \mathrm{Al} \mathrm{CVn}$ & M. Breger et al. \\
\hline & o And. $\mathrm{KX}$ And. $\mathrm{KY}$ & $\begin{array}{l}\text { And, LQ And. EW Lac } \\
\text { C. Stagg }\end{array}$ \\
\hline $\begin{array}{l}17 . \\
18 \mathrm{E}\end{array}$ & $\begin{array}{l}46 \text { Eri, 210G Eri } \\
\alpha \text { Aur, } 9 \text { Aur } \\
\text { HR } 8062 \\
4 \text { Al CVn } \\
\text { HN CMa (HR } 2724\end{array}$ & $\begin{array}{l}\text { J. Manfroid, P. Renson } \\
\text { K. Krisciunas } \\
\text { L. F. Snyder } \\
\text { M. Breger } \\
\text { M. Breger et al. }\end{array}$ \\
\hline
\end{tabular}

E: Data also available by electronic means

\section{GENERAL CATALOG OF VARIABLE STARS (N. N. Samus)}

The third and fourth volumes of the 4th edition of the GCVS were published in 1987 and 1990 respectively. In volumes I - III the information on variable stars in all 88 constellations had been presented. We had hoped to finish the 4th edition within two or three years. but the last three years have unexpectedly been difficult years for the GCVS team: In 1988 Prof. N. N. Kholopov died and furthermore 
the team encountered considerable difficulties in collecting the information on extragalactic variables. especially in connection with positional data.

It was decided to change the order of the remaining volumes of the 4th edition and to publish the reference volume first and the volume with extragalactic data later. During Summer, 1990 volume IV has appeared in print and was distributed to the users. It contains a list of variables in order of their Right Ascension. a list arranged by types of variability, and identification tables enabling the user to identify GCVS and NSV stars with major star catalogues (HD, BD. CoD, CPD. BS, etc.) and with some minor, but useful lists. These identification tables are published for the first time since 1958.

Volume $\mathrm{V}$ will contain information on extragalactic variable stars. We have already prepared data on variables in the Magellanic Clouds, M 31, M 33, and several dwarf galaxies. Equatorial coordinates of variable stars (when not directly available) have been deduced from published rectangular coordinates. but in many cases the team was forced to measure the coordinates itself. In fact, coordinates for several thousand stars in the Magellanic Clouds have been remeasured by Dr. V. P. Goranskij. Volume V will hopefully be finished during 1991.

Work on the name lists has continued. Lists 68 and 69 were published in 1987 and 1989. The new list, number 70 , is nearly completed.

The GCVS team plans to prepare the GCVS data base in computer readable form and to make it available to users. By now the GCVS card catalogue consists of about 500000 cards. each containing data on one star from one publication. The amount of information on the cards is estimated to be about 0.5 Gbyte. The first stage of this work should be a presentation of the data for the last 7 to 10 years on magnetic tape.

\section{Early-Type Variable Stars (Luis A. Balona)}

\section{INTRODUCTION}

The discovery of line profile variability in 53 Per, $\zeta$ Oph and in Be stars such as $\lambda$ Eri has stimulated many observations and much discussion on the nature of the variations. Recent reviews on the line profile variables stars can be found in the proceedings of the following conferences: by Percy in 'The physics of Be stars' (ed. Slettebak. Cambridge, 1987) and by Balona in 'Confrontation between stellar pulsation and evolution' (ed. Cacciari, Bologna, 1990). A review on the B Cep variables by Sterken can also be found in the latter reference. Other conferences where these stars were discussed include 'Impact of very high S/N spectroscopy on stellar physics' (ed. Strobel \& Spite, Kluwer, 1987) and 'Angular momentum and mass loss for hot stars' (ed. Willson \& Bowen. Ames, 1989).

A comprehensive review of the variability of the hottest stars can be found in the NASA/CNRS monograph series 'O. Of and Wolf-Rayet stars' (ed. Conti \& Underhill, 1990). Some discussion on the variability of Wolf-Rayet stars is to be found in 'Physics of luminous blue variables' (ed. Davidson, Moffat \& Lamers, Kluwer. 1988) while Abbot \& Conti (Ann. Rev. Astron. Astrophys. 25, 113, 1987) have presented a valuable review on these stars.

\section{B CEP STARS}

Lee \& Saio (MN 221, 365, 1986; MN 224, 513, 1987: MN 225, 643, 1987: MN 237, 875, 1989; ApJ 349. 570, 1990) have discovered a mechanism for exciting high-order $g$ modes in rotating $B$ stars. However, it is almost certain that many $B$ Cep stars are pulsating in the radial mode, which cannot be excited in this way. It seems that the solution is most likely to be found in an upward revision of envelope opacities (Cox \& Morgan in 'Confrontation between stellar pulsation and evolution,' Bologna, 1990).

Waelkens \& Rufener (AA 201, L5, 1988) found that PHL346, a star lying well above the galactic plane, is a B Cep variable while Sterken \& Jerzykiewicz (MN 235.565. 1988) discovered a possible B Cep candidate in the LMC. These interesting stars offer the possibility of probing the pulsation properties as a function of metallicity. Defining the limits of the instability strip is an important observational task. Progress in this direction was made by Waelkens \& Heynderickx (AA 208. 129. 1989) who reported the discovery of two $B$ Cep variables near the extreme luminosity limits. The less luminous star has a longer than expected period, signifying the possible presence of a g mode.

The identification of pulsation modes in B Cep stars should give clues to the pulsation mechanism and opens up the possibility of asteroseismology. To this end, Aerts. Balona \& Waelkens (in 'Confrontation between stellar pulsation and evolution, Bologna, 1990) have applied a method of Balona (MN 224, 41, 1987) to line profile observations of several bright B Cep stars. 
The case of $\alpha$ Vir, a well-observed B Cep star which stopped pulsating in 1974, is well known. it is possible that 53 Psc might also have behaved in the same way. Reports of $\beta$ Cep type variations were reported as long ago as 1954. Wolf (IBVS 3003, 1987) found it to have a large light amplitude $(0.035$ mag), but subsequent observations by Le Contel et al. (IBVS 3131, 1988) and Balona (IBVS 3157, 1988) did not show any significant variability.

The star $\epsilon$ Per has not been previously classified as a $B$ Cep variable, yet the photometric period and spectral type are typical of this class. The extreme line profile variations have been studied intensively by Smith et al. (ApJ 320. 768, 1987) and most recently by Gies \& Kullajinaya (ApJ 326. 813. 1988). Four periods are present, but because of aliasing problems it is not clear if all of them have been correctly identified. This also poses a problem for mode identification. Gies \& Kullajinaya find that the line profiles are best fitted by modes with azimuthal numbers $m=-3,-4,-5$ and -6 .

\section{PER AND $\zeta$ OPH STARS}

The work of Lee \& Saio (see above) has placed the study of nonradial pulsations (NRP) of hot. massive stars on a firm theoretical foundation. The theory predicts that the superperiod is approximately the rotation period of the convective core. This theory is of particular relevance to the 53 Per and $\zeta$ Oph stars which are thought to be NRPs.

The 53 Per stars are sharp-lined B stars showing long-period line-profile and light variations characteristic of $\mathrm{g}$ modes of low spherical harmonic order. Unfortunately, the poor sampling interval of the observations makes period determination very uncertain. Hence it is very difficult to test the NRP interpretation in the framework of Lee and Saio's theory. Very few observations have been made of these stars in recent years. Chapellier et al. (AA 176, 255, 1987) found short period light variations in ¿. Her which are typical of a $B$ Cep star but not a 53 Per variable. Le Contel et al. (Acta Astron. 39. 227. 1989) observed 53 Per spectroscopically and found that the radial velocity can be modelled with a single sinusoidal period of 2.36 days. It is clear that a concerted effort to determine the periods of these stars needs to be made if we are to understand the nature of the variability. If it turns out that there is really only one underlying period, then it may be possible to model the light and line-profile variations in a way which does not involve NRP. However. Waelkens (in 'Confrontation between stellar pulsation and evolution, ed. Cacciari. Bologna, 1990) has found a beat period with a time scale of many hundreds of days in the light curves of several mid- $B$ stars which could be interpreted as multiperiodicity.

The $\zeta$ Oph stars show line profile variations characteristic of high-order NRP with azimuthal numbers in the range $4 . \mathrm{ml} \cdot 16$. These are observed as low-intensity absorption features moving across the line profile from blue to red (the 'moving bumps'). Most of the known $\zeta$ Oph variables are late $O$ to mid B stars. Baade (AA 222, 200, 1989) looked for, but did not find. moving bumps in 22 late $B$ stars. On the other hand, Walker, Yang \& Fahlmann (ApJ 320. L139, 1987) have discovered moving bumps in two $\delta$ Scuti stars. It is highly probable that the moving bumps in $\delta$ Scuti stars are caused by the same excitation mechanism which is responsible for the low-order line profile and light variations. These stars should therefore not be confused with the O-and B-type $\zeta$ Oph stars for which this mechanism is not applicable.

The observed periods of the $\zeta$ Oph variables are only a few hours, but because of their rapid rotation the periods in the rotating frame are long. In fact, it appears that the superperiod is rather close to the expected rotation period as judged from the projected rotational velocity (Balona 1990. MN 245 , 92). This means that the velocity and surface temperature perturbations are fixed or nearly fixed to the surface of the star. In terms of Lee \& Saio's model, this implies that the rotation period of the core must be close to that of the surface, i.e. that the stars rotate as solid bodies (Balona, MN 245, 92, 1990). This has important consequences for the evolution of B-type stars. Harmanec (Bull. Astron. Inst. Czech. 40. 201. 1989) has interpreted the $\zeta$ Oph phenomenon in terms of a non-uniform surface brightness distribution instead of NRP. His model has the potential for explaining the behaviour of discrete absorption components in the UV resonance lines.

\section{$\lambda$ ERI STARS}

This new classification has been introduced to describe the periodic Be star variables. Owing to photometric surveys by Balona et al. (AA Suppl. 71, 11. 1987). Stagg et al. (MN 234, 1021, 1988) and Cuypers et al. (AA Suppl. 81, 151, 1989), periods of more than 30 stars have been obtained. These stars also show line profile variations which have been ascribed to low-order NRP. Indeed, such variations are a characteristic property of most Be stars, suggesting that it is closely connected to the enhanced mass loss responsible for the circumstellar material. 
A very important contribution to our knowledge of these stars was made by Smith (Ap.J. Suppl. $71,357,1989$ ) who found that there is no correlation between the amplitude of the profile variation and the Be emission level in $\lambda$ Eri. He found that the line profiles are accompanied by various kinds of spectral transients which cannot easily be attributed to beating effects of several NRP modes. Smith attributes these to small-scale magnetic flaring processes.

Balona (MN 245, 92, 1990) analysed the photometric properties of 54 Be stars. Sixty percent of these stars turned out to be $\lambda$ Eri variables with periods in the range 0.5 to 3 days and amplitudes ranging from several millimag to over $0.1 \mathrm{mag}$. About half of the $\lambda$ Eri stars showed double-wave light curves. The light variations in these stars are characterized by irregular fluctuations or flickering with time scales of 0.5 days or longer. The shapes and amplitudes of the light curves vary quite markedly from season to season, but the period remains the same. The most important finding is that there is a strong correlation between the photometric period and the projected rotational velocity. Balona shows that this correlation can only be understood if the photometric and rotational periods do not differ by more than $7 \%$. In terms of the NRP interpretation and the theory of Lee $\&$ Saio, this implies a solid body rotational law. Balona argues that the most plausible interpretation of these results is not NRP but the rotation of a star with a non-uniform surface brightness distribution. This is supported by observations of the Be star $\kappa$ CMa which cannot be explained in terms of a superposition of a few NRP modes.

\section{O STARS, SUPERGIANT VARIABLES AND WOLF-RAYET STARS}

Fullerton (Ph.D. Thesis, University of Toronto 1990) conducted a spectroscopic survey of a sample of $\mathrm{O}$ stars to search for line profile variations. Significant variations with time scales of hours to days were detected in 75 percent of the 32 stars observed. The limited temporal sampling was insufficient for period determination. The cause of the variations is not yet understood, though in the case of HD 34656 radial pulsation seems very likely. The microvariability of many $O$ stars and earlytype supergiants has been studied by van Genderen et al. (AA Suppl. 74, 453, 1988; AA Suppl. $79,263$. 1989). Some stars showed light variations with time scales of several days but in one or two cases the time scale was as short as a few hours. The data are not sufficient to show if any periodicities are present.

A search for periodicity in the light variations of Wolf-Rayet stars has been pursued by Balona. Egan \& Marang (MN 240,103, 1989) who found evidence for multiperiodicity in two stars, WR6 and WR40. These periodicities are unlikely to be coherent but are probably manifestations of the quasiperiodic variations seen in many other WR stars. An important result of this work is that none of the 17 stars surveyed shows evidence of short-period variability typical of NRP. Gosset et al. (MN 238, 97. 1989) found WR40 to pe quasi-periodic with a dominant period of 6.25 days.

\section{4. $\delta$ Scuti and roAp Stars}

(D. W. Kurtz)

\section{$\delta$ SCUTISTARS}

A new, informal publication appeared for the first time in 1989 called the 'Delta Scuti Star Newsletter'. This is currently edited by M. Breger of the University of Vienna and can be obtained free of charge by writing to him. The first two issues have been very interesting with up-to-date notes, papers, discussions of current problems in $\delta$ Scuti star research and observations. Students new to $\delta$ Scuti star research will find the Newsletter useful for learning what other researchers are working on; veteran researchers are encouraged to send notes to the Newsletter publicizing their current research. This is especially important in the case of Eastern European, Soviet, Chinese, and other astronomers who may often publish their work in journals which are not available to the entire community, which have long delays before English translations are available, or which are published in languages other than English.

No new, thorough review of the $\delta$ Scuti stars has appeared since the last of these reports. Kurtz (in 'Multi-Mode Stellar Pulsation. ed. Kovacs et al.. Konkoly Observatory 1988, p. 93) gives a short observational review with good references to previous reviews. Reviews with a more theoretical viewpoint are given by Shibahashi (Lect. Notes Phys. 274. 112) and Dziembowski (Lect. Notes Phys., in press).

$\delta$ Scuti star research was plagued for many years by a dearth of definitive frequency analyses of extensive data sets. In the last few years, however, immense effort has been expended on several multisite campaigns on $\delta$ Scuti stars which have given interesting and definitive results. McNamara et al. (IAU Coll. 111, 276) present preliminary results for a world-wide campaign on $\delta$ Scuti itself; their final 
analysis is still in progress. Michel et al. (Conf. Stellar Puls. \& Evol., in press) have set up an on-going program of multisite observations of $\delta$ Scuti stars; their first results on GX Peg found five frequencies. Breger and his co-workers present multisite campaigns on $\theta^{2}$ Tau and $4 \mathrm{CVn}$. In $\theta^{2}$ Tau Breger et al. (AA 214, 209) found five pulsation frequencies, four of which were found to have the same amplitudes in a previous independent campaign. This sort of observational result should make asteroseismology possible with better theoretical models. Kovacs (IAU Coll. 111, 271) is working on this problem. Dziembowski \& Krolikowska (Acta Astron. 40.19) have looked at mode trapping as a mode selection mechanism. They find trapping in their models for $\ell=1$, but not $\ell=2$ or 3 , and they discuss the implications of this for asteroseismology.

Breger et al. (AA 214, 209) successfully modeled the O-C diagrams of $\theta^{2}$ Tau with light-time effects in its 141-day binary orbit. Light-time effects in the 1181.1-day binary orbit of the large-amplitude $\delta$ Scuti star, SZ Lyn, have also been found by Moffett et al. (AJ 95, 1534) and Paparo et al. (Astrophys. Space Sci. 149. 73); in 78 Tau they have been reported by Kovacs \& Paparo (MN 237, 201).

There has long been dispute about whether $\delta$ Scuti stars pulsate in modes with constant amplitudes, or whether growth and decay times may be short compared to the time-span of the observations so that amplitudes vary on short time scales. This problem has now been solved: Both of these behaviors occur; different stars do different things. Breger et al.'s work on $\theta^{2}$ Tau showed constant frequencies with constant amplitudes. Breger et al. (AA 231. 56) and Breger (Comm. Asteroseis. Vienna. no. 12) find variable amplitudes for $4 \mathrm{CVn}$. They suggest that the long-term amplitude modulation may be similar to the Blazhko Effect in RR Lyr stars, or the amplitude modulation in the unique Cepheid HR 7308. Lampens \& Rufener (AA Suppl. 83, 145) have made extensive observations of 4 known and 6 newly discovered $\delta$ Scuti stars. One of those stars. HR 547. shows amplitude modulation with a period they suggest to be 334 days. They also compare this star with HR 7308. King \& Liu (PASP, 102, 328) have shown from radial velocity studies that $4 \mathrm{CVn}$ is not a binary which it previously had been reported to be; they discuss similar confusion in some other $\delta$ Scuti stars.

Dziembowski et al. (Acta Astron. 38. 61) attacked the problem of the mechanism which limits amplitudes in $\delta$ Scuti stars. They showed that three-mode coupling significantly reduces amplitude for stars with $v \sin i, 20 \mathrm{~km} / \mathrm{sec}$. They pointed out that constant amplitude solutions are unlikely and wondered whether $\delta$ Scuti stars may have variable amplitudes on a long time scale. Breger et al.'s work on $4 \mathrm{CVn}$ shows that they may. Takeuti ( $\delta$ Scuti Newsl. 2. 8) has examined period switching and amplitude modulation in a second-order theory of coupled oscillation.

Period changes have now been reported in some $\delta$ Scuti stars: whether these are evolutionary, caused by light-time effects in binary orbits, or are caused by some other physjcal mechanism is not yet known. Breger reports for the two main periods in $4 \mathrm{CVn} \mathrm{dP} / \mathrm{dt}=-3.4 \times 10^{-1} \mathrm{~d} / \mathrm{d}$ and $-1.4 \times 10^{-9}$ $\mathrm{d} / \mathrm{d}$. These changes are larger than expected and of the wrong sign for evolutionary changes. In $\mathrm{SZ}$ Lyn Moffett et al. find $\mathrm{dP} / \mathrm{dt}=+4 \times 10^{-11} \mathrm{~d} / \mathrm{d}$ : Paparo et al. find $+1.87 \times 10^{-11} \mathrm{~d} / \mathrm{d}$. Rodriguez et al. (Rev. Mex. AA 16, 7) and Jiang (Chin. AA 11, 343) report dP/dt $=+3 \times 10^{-11} \mathrm{~d} / \mathrm{d}$ for AD CMi. Pena et al. (Lect. Notes Phys. 274, 126) suggested $\mathrm{dP} / \mathrm{dt}=-1.5 \times 10^{-11} \mathrm{~d} / \mathrm{d}$ for $\mathrm{CY}$ Aqr and $\mathrm{dP} / \mathrm{dt}=-6.0 \times 10^{-12} \mathrm{~d} / \mathrm{d}$ for DY Peg.

The number of $\delta$ Scuti stars continues to grow as does the literature. Garcia et al. (Bull. Inf. CDS 34) have compiled a very useful catalogue and data base for $\delta$ Scuti stars. It is most important that this data base be maintained; their efforts are appreciated by the entire community.

King (PASP 102, 658) has looked at 72 o Scuti stars observed by IRAS, 37 of which were detected at $12 \mu \mathrm{m}$ and 12 of which were detected at $25 \mu \mathrm{m}$. He found no evidence of excess mass loss compared to normal stars. IR excesses he did find showed no correlation with rotation. He produced a tight $\log \mathrm{P}-\mathrm{M}(12 \mu \mathrm{m})$ diagram, but only after excluding $\delta$ Del stars, IR excess stars, and some others.

Teays et al. (ApJ 343, 916), Pasinetti et al. and Pastori et al. (Conf. Stellar Puls. \& Evol., in press), and Fracassini et al. (AA, in press) have used IUE observations of $\delta$ Scuti stars to study their chromospheric activity. Some $\delta$ Scuti stars show chromospheric activity, some do not. Of those that do, some show maximum activity at pulsation maximum, some at minimum. There is some evidence in the UV observations for mass loss. It would be interesting to look at both IR and UV observations in cases where mass loss is suspected.

Walker \& Yang (Liege Coll. 27, 337), Walker et al. (ApJ 320, L39), and Kennelly et al. (Conf. Stellar Puls. \& Evol., in press) have found travelling bumps in high-precision line profile studies of 4 $\delta$ Scuti stars similar to the features seen in the line profiles of some B star variables. They attribute 
these bumps to high-degree $(\ell, \mathrm{m} " 1)$ non-radial pulsation modes which standard photometric observations cannot detect. The implications of high-degree modes for Dziembowski \& Krolikowska's models of mode trapping have not been examined.

Rachkovskaya and Lyubimkov \& Rachkovskaya (Bull. Crimean Astron. Obs. 76, 3; 75, $137 ; 74$. 14: 73, 76:72,73;71, 128) have done abundance analyses on $\delta$ Scuti stars, most of which are $\delta$ Del stars. They suggest that $20 \mathrm{CVn}, \delta$ Del, V644 Her, 44 Tau, 28 And and HD 127986 all have anomalous abundances. This agrees with some, and contradicts some, previous work; 28 And has been found to have normal abundances in at least two previous studies. Kurtz (MN 238, 1077) found a classical Am star. HD 1097, which is a $\delta$ Scuti star. This, along with the existence of the roAp stars, indicates that extreme abundance anomalies can coexist with low amplitude pulsation in A stars.

Frandsen et al. (AA, 215, 287) could find no $\delta$ Scuti stars in the young clusters Mellotte 105 and NGC 4755, yet they found a dozen in the older cluster NGC 2660. Frandsen \& Kjeldsen (ESA SP-286. 575) present evidence of a changing fraction of pulsators with age. Tsvetkov (Astrophys. Space Sci. 151, 39 and 47) has attempted to determine a period-age relation for $\delta$ Scuti stars and Cepheids; he has then used these relations to try to determine cluster ages.

There has long been a problem matching theoretical and observed period ratios for the fundamental to first overtone pulsation modes in $\delta$ Scuti stars. Andreasen \& Petersen (AA, 192. L4) and Andreasen (AA, 201, 72) have probably solved this problem by making models with opacities 2.5 times higher than the Cox-Stewart opacities. These higher opacities were originally suggested by Simon in 1982 as a solution to the problem of disagreement of evolutionary and pulsational masses in Cepheids and a variety of other problems in pulsating stars. The astronomical grapevine reports that new laboratory opacities are significantly higher than the Cox-Stewart opacities; whether they are 2.5 times higher will not be known until they are published.

Studies of the shapes of Cepheid and RR Lyrae light curves have been put on a quantitative basis for several years now by Fourier decomposition of the light curves. Poretti et al. (AA 228, 350) and Antonello et al. (AA 171, 131) have attempted to do this for high-amplitude $\delta$ Scuti stars; they find an apparent bimodal distribution in the ratio of amplitudes of the fundamental and first-overtone modes. but there is no obvious physical reason for this. Poretti \& Antonello (AA 199, 191) discussed some interesting cases of $\delta$ Scuti stars with descending branches of their light curves steeper than the ascending branches, or with slight stand-stills on the rising branches. This general problem of the form of the non- linearities in the large-amplitude $\delta$ Scuti stars is not well studied.

Many notes and short papers on individual stars have been published in the last three years. The following is not an exhaustive list; updated versions of Garcia et al.'s database is the best source of a complete list. Mantagazza \& Poretti (AA 230, 91) found two periods in HD 16439 and suggested one is due to a nonradial mode. HD 16723, HD 88824, HD 134185, HD 171369, HD 176723, and HD 290764 were discovered to be $\delta$ Scuti stars by Lampens \& Rufener (AA Suppl. 83, 328). Poretti et al. (AA 181, 273) found HD 37819 to be a double mode $\delta$ Scuti star.

Jerzykiewicz \& Sterken (Acta Astron. 40. 117) found two periods in the new $\delta$ Scuti star HD 59594. Oja (AA 184, 215) discovered HD 79889 to be a new high-amplitude $(\Delta V=0.4$ mag) $\delta$ Scuti star. Jiang (Publ. Astron. Inst. Czech. 70, 333; Acta Astron. Sinica 29. 381) suggested that HD 94033 is a binary and that Pop. II $\delta$ Scuti stars have masses in the range 0.6 to 0.9 solar masses and Pop. I $\delta$ Scuti stars have masses of 1.5 to 2.0 solar masses.

HD 147941 was found to be a new $\delta$ Scuti star by Yao \& Tong (IBVS 3334). Mangeney et al. (ESA SP-286) suggest that 63 Her pulsates in the radial fundamental, plus two nonradial $p$ modes, plus probably three g-modes; the latter is important and needs confirmation. Bakos \& Tremko (Publ. Astron. Inst. Czech. 70,67) found AZ CMi is a binary and suggest a radius of 2.6 solar radii with first overtone pulsation. Lopez de Coca et al. (Rev. Mex. AA 15.59) observed 44 Tau and suggested radial mode pulsation. Rolland et al. (Rev. Mex. AA 14, 419) found two periods for $\delta$ Ser. Voroskilov et al. (Astron. Tsirk. 1516) found $\beta$ Cas to be singly periodic. Reid \& Welch (AJ 95, 1510) obtained new observations and did frequency analyses of HR 6391. HR 7222 and HR 7331.

\section{RAPIDLY OSCILLATING Ap (roAp) STARS}

See Kurtz (Ann. Rev. Astron. Astrophys. 28, 607) and Matthews (PASP. in press) for thorough recent reviews of the roAp stars complete to the beginning of 1990 . Martinez \& Kurtz (IBVS. in press) have begun a program to find new roAp stars and report five new ones from their initial results: HD 161459. HD 193756. HD 190290. HD 196470 and HD 218495. Verheest et al. (MN 245, 392) looked at the possibility of chaotic pulsation in roAp stars. 


\section{Cepheids \\ (Edward. G. Schmidt)}

\section{INTRODUCTION}

This survey covers studies published between July 1. 1987 and June 30. 1990. During that interval. the proceedings of a conference on pulsating stars entitled 'The Use of Pulsating Stars in Fundamental Problems of Astronomy, IAU Coll. 111' (1989, Cambridge University Press, ed. E. Schmidt) appeared and a conference was held in Bologna in May 1990 devoted to pulsating stars.

\section{FUNDAMENTAL DATA}

Simon (1990. Bologna Conference) reviewed the impact of fundamental Cepheid data on the theories of stellar pulsation and evolution. He has also reviewed the application of Fourier decomposition to the analysis of light and velocity curves (in 'Pulsation and Mass loss in Stars,' eds. Stalio \& Willson. Reidel, 1988).

During the past few years new velocity curves have been published by Barnes et al. (ApJ Suppl. 65. 307, 1987; ApJ Suppl. 66, 43, 1988) and Wilson et al. (ApJ Suppl. 69, 951, 1989) while Moffett \& Barnes (PASP 99. 1206, 1987) have tabulated mean velocities for many stars. Velocities for Cepheids in the Magellanic clouds are even becoming available (Imbert et al. 1989. AA Suppl. 81, 339).

Fernie (AJ 94, 1003, 1987) and Spencer Jones (MN 238, 269, 1989) discussed the question of Cepheid reddenings. Although problems remain, it appears that agreement among various methods is improving. Fernie (ApJ Suppl. 72, 153) has tabulated the color excesses for 328 Cepheids on a uniform scale and has summarized the statistical properties of stars within the instability strip (ApJ 354, 295. 1990).

The determinations of effective temperatures and abundances have received relatively little attention. Fernley et al. (MN 237. 947. 1989) used the infrared flux method to determine effective temperatures while Coker at al. (PASP 101, 594, 1989) used energy distributions throughout the visual region. Raga et al. (ApJ 347, 1107, 1989) investigated the helium abundance in the type II Cepheid W Vir using emission-line strengths.

There has been considerable activity in the area of radius determinations for Cepheids. Moffett \& Barnes (ApJ 323. 280. 1987). Turner (AJ 96, 1565. 1988), Coker et al. (PASP 101, 594. 1989), Gieren et al. (ApJ 342, 467, 1989), Fernley et al. (MN 237, 947, 1989), and Coulson \& Caldwell (MN $240,285$. 1989) have provided lists of radii determined from various versions of the Baade-Wesselink method.

Moffett (IAU Coll. 111, 1989) reviewed the various versions of the Baade-Wesselink method. Uncertainties in radius determinations have been discussed by the above authors as well as Sasselov et al. (ApJ 337. L29. 1989) and Bohm-Vitense et al. (ApJ 343, 343, 1989) while Simon (PASP 99, 868. 1987; MN 237, 163, 1989) has proposed an inversion of the usual Baade-Wesselink method. It appears that Cepheid radii are becoming more reliable and the differences among various investigators are becoming smaller.

\section{INDIVIDUAL STARS}

Following the suggestion that the two-day Cepheid EW Tau had a peculiar overtone period (Gieren \& Matthews 1987, AJ 94, 431), two groups obtained further data (Fernie 1987, PASP 99, 1093; Gieren et al. 1989. AJ 98, 1672), and Gieren et al. (AJ 99, 1196, 1990) discussed the pulsation based on all available data. It now appears that there is nothing unusual about this star aside from its being an overtone pulsator. Mavridis et al. (AA Suppl. 80, 279, 1989) obtained extensive observations of V1162 Aql, while van Genderen \& Nithardjo (AA 221, 230, 1989) obtained photometry for the newly discovered 74-day Cepheid HDE 270100 in the LMC. Kardopolov \& Filip'ev (A. Zh. 65, 816. 1988) discovered a new Cepheid and a star which may be a new UU Her star among stars previously classified as ls variables.

\section{CEPHEIDS IN CLUSTERS AND ASSOCIATIONS}

The emphasis in this area has been on studies of clusters and associations previously known or thought to contain Cepheids. Mermilliod et al. (AA Suppl. 70, 389, 1987) obtained velocities of stars in eight clusters and confirmed seven of the Cepheids as members while Harris et al. (AJ 94, 403. 1987) used radial velocities to confirm the membership of DL Cas in NGC 129. Gieren (PASP 100, 262. 1988) compared the distances of nine Cepheids obtained from the surface brightness method with cluster and association distances and confirmed membership for seven of the Cepheids. Mateo \& Madore (PASP 100. 1222, 1988) gave a preliminary report on deep photometry of four clusters with Cepheids aimed at 
better defining the main sequences while Walker (MN 229, 31, 1987) presented photometry of faint stars in Ruprecht 79 which contains CS Vel.

Pavlovskaya \& Filippova (A. Zh. 65, 907, 1988). Eigenson et al. (A. Zh. 65, 1244, 1988) and Berdnikov and Efremov (A. Zh, 66, 537, 1988) have sought to establish the existence of much larger groupings of Cepheids with presumably common origin. Karimova (Pis. A. Zh. 15, 777, 1989) has found such groupings in the LMC. Pavlovskaya \& Filippova (A. Zh. 66, 1165, 1989) have compared the groupings of Cepheids with those of giant stars and star clusters.

\section{THE PERIOD-LUMINOSITY RELATION}

The calibration of the period-luminosity $(\mathrm{PL})$ relation has continued to receive considerable attention. Feast \& Walker (Ann. Rev. Astron. Astrophys. 25, 345, 1987) reviewed in detail the calibration of both the PL and PLC relations while Rowan-Robinson (Space Sci. Rev. 48, 1. 1988) reviewed the calibration in the context of the extragalactic distance scale. Hindsley \& Bell (ApJ 341, 1004, 1989; ApJ $348,673,1990$ ) used surface brightnesses from model atmospheres to perform a Baade-Wesselink analysis from which they derived the absolute magnitudes of 23 Cepheids. They then produced PL relations for the visual and infrared bands. Gieren (ApJ 329, 790, 1988) used absolute magnitudes for 52 Cepheids obtained from the surface brightness method to derive a PL relation while Barnes \& Moffett (1990. Bologna Conference) extended this result by including 101 Cepheids. Gieren compared these results with previous calibrations from clusters and found that the various methods seem to be converging. Visvanathan (ApJ 346, 629, 1989) used cluster Cepheids to calibrate the PL relation for his infrared IV band.

Stothers (ApJ 329, 712, 1988) carried out a theoretical study of the effects of elemental abundance on the PL and PLC relations. He found that the PLC relation is rather sensitive to metallicity while the sensitivity of the PL relation is negligible. Opolsky (Acta Astr. 38, 375, 1988) pointed out that the color term in the PLC relation nearly cancels the effects of reddening. Exploiting this fact he derived a relation between distance, $V$ magnitude, $B-V$ and period which he applied to a study of the space distribution of Cepheids. The application of various combinations of color and magnitude to distance determination have been discussed by Freedman (ApJ 326. 691. 1988). Walker (ApJ 332, L37. 1988) and Stift (AA 229, 143, 1990).

\section{BINARY CEPHEIDS}

The study of Cepheids in binary systems has attracted much interest in recent years. Spectroscopic orbits have now been derived for the Cepheids U Aql (Lyons et al. 1987. PASP 99, 610), FF Aq! (AJ 99, 1598, 1990), SU Cyg (Evans 1988, ApJ Suppl. 66, 343), S Mus (Evans 1990, PASP 102. 551), AW Per (Evans 1989, AJ 97, 1153). S Sge (IAU Coll. 111, 1989) and W Sgr (Babel et al. 1989. AA 216. 125). Szabados (MN 242, 285, 1990) determined orbital periods for XX Cen, $X$ Sgr and V350 Sgr; and Gieren (AA 216, 135. 1989) showed that the Cepheid T Mon has too long a period to be useful for mass determination.

Although none of these binary systems has yet yielded a definitive mass. Evans \& Bolton (ApJ 356. 630.1990) argued that the mass of SU Cyg lies between 5.9 and 6.2 solar masses based on a study of the companions. This agrees with evolutionary calculations. Bohm-Vitense et al. (AJ 99, 353, 1990), on the other hand, derived a mass of 4.6 to 6.1 solar masses for S Mus which is significantly less than the evolutionary mass of 7.5 solar masses. More work is required to resolve this apparent conflict.

Evans (PASP 100.724, 1988) was unable to detect the companion of Polaris in the ultraviolet and concluded that it must be a main sequence star later than $A 8$ or a white dwart. On the other hand, for AW Per she (AJ 97, 1737, 1989) was able to detect the companion spectrum and show that it is an early type star. In this case, the companion itself seems to be a binary on the basis of its mass and luminosity.

Szabados (PASP 100, 589, 1988) demonstrated light time effects in FN Aql and RX Aur which indicate that they are binaries while Gieren (PASP 101,160,1989) found evidence from radial velocity data that $Z$ Lac is a long period binary. Mermilliod et al. (AA Suppl. 70. 389. 1987) and Harris et al. (AJ 94. 403. 1987) found that DL Cas in NGC 129 is a spectroscopic binary.

Opal et al. (AJ 96, 1677, 1988) obtained CCD images of the double Cepheid CE Cas in the cluster NGC 7790 and separated the light curves of the two components.

\section{PULSATIONAL PROPERTIES}

Buchler et al. (ApJ 351.617, 1990) constructed a series of hydrodynamic models to study the $\mathrm{P}_{2} / \mathrm{P}_{\mathrm{o}}$ resonance near ten days. Kovacs \& Buchler (ApJ 346. 898, 1989) applied the amplitude equation 
formalism to this series of models and showed that the period resonance is clearly the cause of the Hertzsprung bump sequence. Kovacs et al. (ApJ 351,606, 1990) then compared the Fourier coefficients from the velocity curves of 57 Cepheids with those from the models and found relatively good agreement. Petersen (AA 226, 151, 1989) calculated periods for model sequences representing classical and type II Cepheids with the purpose of searching for other period resonances which might play a role in the shape of light and velocity curves. Andreasen (AA 191, 71, 1988; AA 196, 159, 1988) analyzed the light curves of galactic and SMC Cepheids. He found that the Fourier diagrams are very similar except that the resonance features occur at slightly longer period in the SMC.

Following a suggestion by Simon (ApJ 260. L87. 1982) interior opacities have been scrutinized as a possible source of error in the results of pulsation theory. Andreasen \& Petersen (AA 192, L4, 1988) and Andreasen (AA 201, 72, 1988) showed that augmenting the opacities in a restricted temperature range can alleviate the mass discrepancy for the beat and bump Cepeids. Carson and Stothers (ApJ 328. 196. 1988) also argued that using the alternate Carson opacities improve the agreement between theory and observation. Some support is given for these suggestions from new opacity studies (Rozsnyai 1989. ApJ 341, 414). On the other hand, Morgan \& Cox (Bologna Conference. 1990) dispute the idea that opacity enhancement will remedy the mass problems entirely. The matter should be settled by new large scale opacity calculations now under way (Mihalas 1989. IAU Coll. 111).

Aikawa et al. (AA 181, 25. 1987) discussed the pulsation mode of SU Cas using both observational data and new models. They concluded it is not a fundamental pulsator but were unable to arrive at a completely satisfactory model. Babel \& Burki (AA 181, 34, 1987) obtained new velocity and light curve data for CO Aur and discussed its pulsation. They conclude that it is pulsating in the first and second overtone in agreement with earlier work.

Buchler and collaborators (ApJ 320, L57, 1987; ApJ 334, 971, 1988; ApJ 355, 590, 1990) have constructed pulsational models which show evidence of chaotic behavior. Based on these models they suggest that the alternating cycles seen in RV Tau stars are due to period doubling as chaos is approached.

\section{MASSES AND EVOLUTION}

Cox (IAU Coll. 111, 1989) reviewed the status of Cepheid mass determinations. Gieren (AA 225. 381, 1989) compared masses determined from Wesselink radii for 101 stars with evolutionary masses. pulsational masses and theoretical masses. He argued that the discrepancies now remaining among these various estimates are within the uncertainties and no new mechanisms need be invoked to explain them.

Deasy (MN 231, 673, 1988) searched for evidence of mass loss in classical Cepheids using infrared data from IRAS and emission line profiles for the $\mathrm{Mg} \mathrm{h}$ and $\mathrm{k}$ lines. He estimated mass loss rates in the range from $10^{-10}$ to $10^{-6}$ solar masses per year although the higher values were relatively unusual. Welch \& Duric (AJ 95, 1794, 1988) failed to detect five classical Cepheids and two type II Cepheids at 5 $\mathrm{GHz}$ with the VLA. This places upper limits on the outflow of ionized gas from these stars between $10^{-9}$ and $10^{-7}$ solar masses per year. These rates are too low to allow mass loss in the instabilty strip to have significant effect on the evolution. In particular, it does not appear that pulsational mass loss can cause stars to become trapped in the instability strip as suggested by Willson \& Bowen (Nature 312, 429 . 1984).

Dinshaw et al. (AJ 98, 2249, 1989) have obtained new velocity data for Polaris and rediscussed its decreasing amplitude and period changes. They conclude that it is leaving the instability strip and pulsation should cease about 1995.

Chiosi (IAU Coll. 111, 1989: PASP 102, 412, 1990) reviewed the status of stellar evolution as it pertains to Cepheids. He emphasized the importance of convective overshoot in reproducing some observational data including the period-frequency distribution but showed that observed rates of mass loss are too low to have an appreciable effect. On the other hand. Li \& Huang (AA 229. 469, 1990) evolved models into the instability with and without mass loss. Using the maximum of the growth rate as an indicator of the center of the instability strip, they find that although stars with mass loss appear lower in luminosity, the position of the strip is unaffected.

\section{TYPE ॥ CEPHEIDS}

Harris and Welch (AJ 98, 981, 1989) studied the orbits of two binary type II Cepheids. They point out that in all three type II Cepheids with known orbits the size of the orbit is small compared with the star implying that mass transfer has played an important role in these systems. 
Alexander et al. (PASP 99, 645, 1987) carried out a photometric study of the prototype star BL Her. They derived a mass for of 0.75 solar masses. Loomis et al. (MN 235, 1059, 1988) obtained light curves for seven possible type II Cepheids which had not been well observed in the past. Of the seven, only two were confirmed as type II Cepheids.

\section{RELATED STARS}

Observational monitoring of several UU Her stars has continued (Sasselov et al. 1987. PASP 99. 967; Fernie 1989. PASP 101, 171; Fernie 1990. PASP 102, 442) with the goal of clarifying their long term behavior. Based on a spectroscopic study Klochkova \& Panchuk (Pis. A. Zh. 15, 617, 1989) suggest that UU Her is in a post-asymptotic giant branch stage of evolution. On the other hand. Fernie \& Sasselov (PASP 101, 513, 1989) showed that the color and period changes are inconsistent with this interpretation while Sasselov et al. (1987 op. cit.) showed that the stars evolve too slowly to be considered as preplanetary nebula objects.

\section{EXTRAGALACTIC CEPHEIDS}

The Cepheids in the Magellanic Clouds continued to attract considerable attention. Welch et al. (ApJ 321, 162, 1987) and Mathewson et al. (ApJ 333, 617, 1988) have used Cepheids to study the structure of the Magellanic Clouds. These two investigations do not. however, agree on the structure of the SMC with the former finding a line of sight depth for the SMC of less than $5 \mathrm{kpc}$ while the latter claims a depth of about $20 \mathrm{kpc}$ in agreement with earlier work.

The discovery of Cepheids in rich Magellanic Cloud clusters is of interest in connection with their evolution. Storm et al. (AA 190, L18, 1988) have identified 17 suspected Cepheids in the LMC cluster NGC 1866 while Mateo et al. (ApJ 353, L11, 1990) have found three new Cepheids in NGC 2157 in the LMC.

Andreasen (AA 186, 159, 1987) searched for double mode pulsation among LMC Cepheids using existing photographic light curves. The dozen possibilities he found are the first reported double mode pulsators in the Magellanic Clouds. However. he points out that there should be many more based on their prevalence in the Milky Way.

The current status and future of the application of Cepheids to the extragalactic distance problem was reviewed by Mould (IAU Coll. 111, 1989). New Cepheid photometry and distances have been obtained for the galaxies NGC 300 (d.m. = 26.35, Madore et al. 1987, ApJ 320, 26), IC 1613 (d.m. = 24.3, Freedman 1988. ApJ 326, 691). NGC 2403 (d.m. = 27.51. Freedman \& Madore 1988. ApJ 332, L63) and M81 (d.m. $=27.59$, Freedman \& Madore 1988, op cit.). Feast (Observatory 108, 119, 1988) discussed the $\mathrm{PL}$ relation in different wavelength bands and combined the results of various photometric studies from the literature to obtain a best estimate for the M33 distance modulus of 24.4.

Sandage (PASP 100, 935, 1988) discussed the effects of a magnitude cutoff on the distances to galaxies determined from Cepheids and derived corrected distances to a number of galaxies. Walker (PASP 100, 949, 1988) corrected Graham's photographic sequences in NGC 300 and derived a larger distance modulus in agreement with that of Madore et al. (1988 op cit.). Metcalfe \& Shanks (PASP 100. 1222. 1988) are checking the BV sequences in a number of nearby galaxies to correct earlier photographic photometry.

Caldwell et al. (PASP 100, 1217, 1988) are searching several galaxies for new Cepheids while Carlson \& Sandage (ApJ 352, 587, 1990) have derived periods and light curves for short period Cepheids in IC 1613 which have not been previously studied. Schmidt \& Spear (MN 236, 567, 1989) and Schmidt (IAU Coll. 111, 1989) obtained light curves for Cepheids in NGC 6822 for comparison with stars in the Milky Way. Although the sample was too small for definitive conclusions, some indication of differences from Milky Way Cepheids were found.

\section{RR Lyrae Stars in the Field}

\section{(T. G. Barnes III)}

This survey of the field RR Lyrae variables covers the period from the last report through August 1990. Because of page limitations not every paper can be cited. Additional citations to RR Lyrae research are contained in the sections of this report which discuss pulsation theory and variable stars in globular clusters and related systems.

\section{CONFERENCES AND REVIEWS}

The following conferences, which included RR Lyrae research, took place or appeared in print during the interval covered by this report: 'Stellar Pulsation.' (Proc. of a conf. held as a memorial to John P. 
Cox at the Los Alamos National Laboratory. Los Alamos. New Mexico. USA, Aug 11 - 15. 1986. Lect. Notes Phys. 274, 1987, eds. Cox et al.); Proc. of the 19th All-Union Conf. of Var. Star Investigators devoted to Prof. V. P. Tsesevich's Memory, Odessa, USSR, 13 - 17 April 1987. Per. Zv. 22, 815, 1988; 'The second conf. on faint blue stars.' (IAU Coll. 95. Tucson. Arizona, USA, 1 - 5 June 1987, eds. Philip et al. 1988); 'Multi-mode stellar puls.' (Proc. of a Workshop. Budapest. Hungary, 1 - 3 Sep 1987, eds. Kovacs et al., 1988); 'The uses of pulsating stars in fund. prob. of Astron..' (IAU Coll. 111. Lincoln. Nebraska. USA. 15 17 Aug 1988, ed. Schmidt); 'Confrontation between Stellar Puls. and Evol.; (Bologna, Italy, May 28 - 31. 1990. ASP Conf. Ser., eds. Cacciari \& Clementini, in press). p. 218)

The Stellar Puls. conf. contains a review of the field RR Lyrae variables by Lub (Stellar Puls. Conf.,

\section{ABSOLUTE MAGNITUDES AND DISTANCES}

The absolute magnitudes of RR Lyrae variables have been an area of intense interest during the last three years. Jones and collaborators applied a modified Baade-Wesselink method to DH Peg (Jones et al. 1988. ApJ 326, 312); RS Boo. TW Her. VY Ser, and UU Vir (Jones et al. 1988, ApJ 332. 206). Further discussion of the SW Dra results is given by Jones (ApJ 326, 305, 1988). These and previous results are discussed by Jones et al. (IAU Symp. 126, 589. 1988). They interpreted their results as showing no dependence of absolute magnitude upon metallicity, and suggested that $M_{v}=0.85$ mag.

Cacciari and collaborators have applied another version of the B-W method to SW And. SW Dra. and SS For (Cacciari et al. 1989. AA 209, 141); YZ Cap. RV Phe, and V440 Sgr (Cacciari et al. 1989. AA 209, 154). The data for these papers were published in Cacciari et al. (AA Suppl. 69, 135, 1987). Their work was summarized by Cacciari et al. (IAU Symp. 126, 587, 1988). Combining all the B-W results previous to, and including, their work, they propose the relation: $M_{v}=1.0+0.17[\mathrm{Fe} / \mathrm{H}] \mathrm{mag}$.

Liu \& Janes have determined distances to thirteen RR Lyrae variables through yet a third B-W method (in 'Calibr. of stellar ages.' Van Vleck Obs., Wesleyan Univ.. Middletown. USA, p. 141. 1988; ApJ Suppl. 69, 593, 1989; ApJ 354, 273, 1990). They found the following relation for the absolute magnitude: $M_{v}=1.05+0.20[\mathrm{Fe} / \mathrm{H}]$ mag. Liu \& Janes have applied the same technique to four RR Lyrae variables in M4 and show that these stars fit their field $\mathrm{M}_{\mathrm{v}}-[\mathrm{Fe} / \mathrm{H}]$ relation (ApJ 360, 561, 1990).

Fernley and collaborators have devised an infrared version of the B-W method and applied it to RR Lyrae variables (Fernley et al., Stellar Puls. conf, p. 239, 1987) including X Ari (Fernley et al. 1989, MN 236, 447). DX Del (Skillen et al. 1990, MN 241, 281) and the RRc variable DH Peg (Fernley et al. 1990, MN 242, 685). Their results for the two RRab variables are consistent with the Cacciari et al. and the Liu \& Janes $\mathrm{M}_{\mathrm{V}}-[\mathrm{Fe} / \mathrm{H}]$ relations.

In related work, problems with the B-W method for RR Lyrae variables have been discussed by Hawley et al. (1987. Stellar Puls. conf, p. 235). Simon (MN 237, 163, 1989) has applied his inversion of the B-W method to SW Dra and X Ari. He was able to determine a radius for SW Dra of 5.3 solar radii, but no solution for $X$ Ari was possible.

A maximum likelihood solution for the mean absolute magnitude of RR Lyrae type ab stars by Zhao (Sci. Sin. 31. 734, 1988) gave $\left(M_{v}\right.$ ' = $0.62 \pm 0.12$ mag.

Noble \& Dickens (in 'New ideas in Astron.' Proc. of a conf. held in honor of the 60th birthday of Halton C. Arp at Venice, Italy, 5 - 7 May 1987, p. 59, 1988) determined the distances to ten globular clusters by main sequence fitting to subdwarfs and thereby found RR Lyrae absolute magnitudes for the variables in the clusters: $M_{v}=0.591+0.126[\mathrm{Fe} / \mathrm{H}] \mathrm{mag}$.

\section{METAL ABUNDANCES}

The relationship between RR Lyrae absolute magnitudes and their metallicities is still a matter of contention among researchers as can be seen from the above absolute magnitude results. In addition to the works cited above, this question is discussed by Fernley et al. (MN 226, 927, 1987) and by Sandage (IAU Coll. 111, 121, 1989; ApJ 350, 603, 1990; ApJ 350, 631, 1990).

A new calibration of phase corrections to the $\Delta S$ parameter has been determined by $S$ mith (PASP 98, 1317, 1987). Zajkova \& Romanov (Per. Zv. 22, 905, 1988) have proposed a revision of the $\Delta S$ parameter. Smith has reported new $\Delta S$ measures for twenty RR Lyrae variables (PASP 102. 124, 1990). and Mendes de Oliveira \& Smith have added eight more $\triangle S$ values (PASP 102, 653, 1990).

Further comments on the relationship of observed properties of RR Lyrae stars to metallicity are included in the section on variable stars in globular clusters. 


\section{RADIAL VELOCITIES}

A large quantity of very high quality radial velocities have become available for RR Lyrae variables in the last few years. Most of these radial velocities were acquired in order to perform BaadeWesselink solutions and the citations are included in the section above on absolute magnitudes. Several additional publications of radial velocities are the following: Barnes et al. gave new velocities for seven variables (ApJ Suppl. 67. 403, 1988). Wilson et ai. reported velocities for eight RR Lyrae stars (ApJ Suppl. 69. 951, 1989).

Saha \& White (PASP 102, $148 \& 495,1990$ ) have suggested that TU UMa is a binary of period 7375 days on the basis of new radial velocities and an analysis of times of maximum light.

\section{MAGNETIC FIELDS, BLAZHKO EFFECT, MULTI-MODE BEHAVIOR}

Romanov et al. (Pis'ma Astr. Zh. 13, 69, 1987; Proc. of the intern. meeting on 'Phys. and evol. of stars'. held in Nizhnij Arkhyz. USSR. $12-17$ October 1987, p. 51) have studied the variation of magnetic field strength in RR Lyrae with respect to the pulsation phase and the Blazhko phase. They discuss the latter dependence in terms of an oblique rotator model.

Gloria (PASP 102, 338, 1990) has analyzed the relationship between the presence of the Blazhko effect and the length of the pulsation period for RRab stars. She found that the stars with Blazhko effect have a significantly shorter mean period than do the stars with non-variable period.

Several researchers have discussed multi-mode behavior as the origin of the light amplitude variations in the Blazhko cycle. Moskalik (Acta Astr. 36. 333. 1987) examined the theory of amplitude modulation due to internal resonances and proposed a 2:1 resonance between the fundamental and the 3rd overtone as the most likely cause of the amplitude variations. Goranskij (Per. Zv. 22. 913, 1988: Astron Zh. 66. 84. 1989) interpreted the variable amplitude of AH Cam in the same way. Additional discussions of multi-mode oscillations in RR Lyrae variables have been given by Kanyo (Multi-mode Stellar Puls. cont., p. 67. 1988), by Szeidl (Multi-mode Stellar Puls. conf., p. 45, 1988) and for ST CVn by Peniche et al. (AA 209, 59. 1989). The star RU Psc was studied for multi-mode behavior by Mendes de Oliveira \& Nemec (PASP 100, 217, 1988). They concluded that the light curve peculiarities are a result of rapid and irregular period changes and not of secondary periodicities.

\section{SHOCK WAVES}

Gillet (IAU Symp. 132. 143, 1988). Gillet \& Crowe (AA 199, 242, 1988) and Gillet et al. (AA 225 445. 1989) have used high spectral resolution to investigate the behavior of $\mathrm{H} \alpha$ with pulsation phase in RR Lyr, X Ari and RX Eri. They interpreted the emission line features in terms of Hill's non-linear hydrodynamic model of shocks which predicted two shocks at two different phases, as observed. In addition. they argued that the shock velocities must be of order 60 and $50 \mathrm{~km} / \mathrm{s}$, which is both supersonic and much larger than the velocity deduced from the radial velocity curves.

\section{RR LYRAE VARIABLES IN SPECIAL FIELDS}

Photometric data have been published on thirteen RR Lyrae variables in Baade's NGC 6522 window (Mack \& Walker 1987, South Afr. Astron. Obs. Circ. 11, 121). Wesselink et al. (ESO Conf. Workshop Proc. 27, 185, 1987) studied a selection of RR Lyrae variables in Plaut's field 3. Ciardullo et al. have discovered an RRab variable projected in front of the Virgo Cluster at a distance of $50 \mathrm{kpc}$ from the Galactic center (AJ, 98, 1648, 1989).

RR Lyrae variables in the halo of M31 have been studied by Pritchet \& van den Bergh (ApJ 316. 517. 1987). They interpreted the observations as showing these variables to belong to Oosterhoff type 1 . A review of RR Lyrae variables in extragalactic systems was given by Pritchet (ASP Conf. Ser. 4, 59. 1988).

\section{LIGHT CURVES, REDDENING, PHOTOMETRIC DATA}

Fourier coefficients derived from the decomposition of pulsating star light curves were intercompared for several classes of variables including RR Lyrae stars by Antonello et al. (AA 171. 131. 1987). A regular decrease in the Fourier decomposition parameter $\phi_{21}$ with decreasing metal abundance has been found by Simon (ApJ 328, 747, 1988) for six RRab variables, which he interprets an opacity effect. Schmidt et al. (ApJ 360,604, 1990) obtained data for 16 variables with periods less than one day. Their Fourier decomposition does not show a clear distinction between the long-period RR Lyrae stars and the anomalous Cepheids. 
Sperauskas (Vilniaus Astr. Obs. Biul. 76, 3, 1987) has discussed the utility of the Vilnius sevencolor photometric system for identifying RR Lyrae variables and other horizontal branch stars. The same author has shown that the Vilnius system can be used to determine reddenings for RR Lyrae variables (Vilniaus Astr. Obs. Biul. 79, 36, 1987). Kadla et al. (Astr. Tsirk. 1503, 3, 1987) have also devised a method to determine reddenings based on UBV photometry and Kurucz's models. They applied the technique to obtain reddenings of thirteen RRc variables (Astr. Tsirk., 1521. 7, 1987).

Large numbers of RR Lyrae variables have had elements determined or revised in the last three years. The results have been published in BAV Rundbrief, IBVS, MVS, GEOS Circ, Astr. Tsirk., J. AAVSO, Per. Zv., and J. British Astron. Assoc. These sources also include references to period variations, to newly discovered RR Lyrae variables and to stars with newly discovered Blazhko effect. Belserene and collaborators (1990, preprint) reported on a study of 29 variables for period changes. About half showed no detected period change and about half had slowly increasing period, consistent with stellar evolution calculations.

\title{
CATALOGUES
}

Heck (Bull. Centre Donnees Stell. 34, 133, 1988) has compiled a new edition of the 1977 bibliographical catalogue of RR Lyrae variables. There are 6367 RR Lyrae variables listed in the catalogue. The new catalogue is available from the CDS in printed and in magnetic format.

Ephemerides for RR Lyrae variables were published for 1988 by Firmaniuk \& Kreiner (Rocznik Astr. Obs. Krakow. Intern. Suppl. 59, 115. 1987) and for 1989 by Firmaniuk et al. (1988. Rocznik Astr. Obs. Krakow. Intern. Suppl. 60, 115, 1988) and also by the AAVSO. Firmaniuk et al. (Astr. Tsirk. 1508, 7, 1987) published a supplement to their catalogue of elements of RR Lyrae stars

\section{Variable Stars in Globular Clusters and Related Systems}

\author{
(Amelia Wehiau)
}

Although an effort has been made to be comprehensive, due to severe space limitations not all references are included. The reader is referred to the Comm. 38 report on globular cluster research and to the following conference proceedings: IAU Coll. 95. Second Conference on Faint Blue Stars, eds. Phillip et al.. 1987: 'Stellar Pulsation.' Lect. Notes Phys. 274, eds. Cox et al., 1987: ESO Workshop No. 27 on 'Stellar Evolution and Dynamics in the Outer Halo;' eds. Azzopardi \& Matteucci, 1987; IAU Symp. 126. The Harlow Shapley Symp. on Globular Cluster Systems, eds. Grindley \& Phillip. 1988; IAU Coll. 111. 'The Use of Pulsating Stars in Fundamental Problems of Astronomy.' ed. Schmidt, 1989; 'Confrontion Between Stellar Pulsation And Evolution,' ASP Conf. Series, eds. Cacciari \& Clementini, 1990.

\section{STUDIES OF PULSATING VARIABLES IN GALACTIC GLOBULAR CLUSTERS}

Listed in order of IAU cluster designation. In addition, data for red variables in 13 clusters is given by Frogel \& Elias (ApJ 324, 823. 1988). NOTE: CS below refers to Costar \& Smith (AJ 96. 1925. 1988).

C0021-723 (NGC 104, 47 TuC): The $\triangle S$ metal-abundance index for V12 is given by CS. IRAS observations by Gillett et al. (AJ 96. 116, 1988) include V3 and V11.

C0050-268 (NGC 288): The $\triangle S$ metal-abundance index for $V 2$ is given by CS

C0512-400 (NGC 1851): Ca abundances obtained by Rodgers \& Harding (PASP 99, 961, 1987) are listed for V10=Stetson $\# 190, V 22=\# 55, V 23=\# 159$ and V26= $\# 340$.

C0734+390 (NGC 2419): Observations of V12 are included in a paper on CCD photometry of the cluster by Christian \& Heasley (AJ 95, 1422, 1988).

C0911-646 (NGC 2808): Three new variables are announced in a study of all known variables in the cluster by Clement \& Hazen (AJ 97, 414, 1989).

C1015-461 (NGC 3201): Calcium abundances from spectrophotometry by Rodgers \& Harding (PASP 101. 40.1989 ) of $18 \mathrm{HB}$ stars include those for V2O and V40.

C1236-264 (NGC 4590, M68): Clement (AJ 99, 240, 1990) has found a double-mode pulsation mass of 0.64 for V3, one of at least 7 double-mode pulsators. V2 and V4 are included in CCD photometry by Alcaino et al. (AJ 99, 1831, 1990).

C1323-472 (NGC 5139, $\omega$ Cen): Dickens (IAU Coll. 111, 141, 1989) discusses the significance of studies of this unusual cluster and gives new results based on photometry of its RR Lyrae stars. Caputo (AA 189. 70. 1988) examines the period-shift versus metallicity relation for RR Lyrae stars in this cluster.

C1339+286 (NGC 5272. M3): A search for double-mode pulsation among 46 variables by Nemec \& 
Clement (AJ 98, 860. 1989) has turned up only V68 and V87, both already known to be RRd stars. Meinunger presents photometry of V38 and variables 41 through 60 (Mitt. Ver. St. 11, pp. 81, 83, 109, 1988: pp. 179, 182, 1989).

C1403+287 (NGC 5466): CCD observations of 17 previously known variables and 9 newly discovered variable blue stragglers are discussed by Harris et al. (CCD's in Astronomy ll, in press).

$\mathrm{C} 1513+000(\mathrm{Pal} 5)$ : CS give $\Delta S$ values for $\mathrm{V} 1$ and $V 5$.

C1514-208 (NGC 5897): Wehlau (AJ 99, 250. 1990) presents light curves and colors for the 8 known variables and discusses their unusual period distribution. $\triangle S$ values for $V 1, V 3$ and $V 6$ are given by $C S$.

C1516+O22 (NGC 5904, M5): Geraschenko et al. (IBVS 3044, 1987; Astr. Tsirk. 1502, 7, 1987) announce 11 new variables, 10 of which are included in a list and finding chart for 27 new variables given by Kravtsov (Astr. Tsirk. 1526, 6. 1988). Values of $\Delta S$ are given for V75 by CS. Cardelli (AJ 98. 324, 1989) discusses V42 and V84 in a paper on the nature of RV Tauri stars. The Baade-Wesselink method is applied to V8 by Carney et al. (IAU Coll. 111, 253, 1989).

C1614-228 (NGC 6093, M80): Wehlau et al. (AJ 99. 1159, 1990) present light curves and colors for the 8 known cluster variables and two nearby Mira stars.

C1620-264 (NGC 6121. M4): An astrometric \& photometric study by Cudworth \& Rees (AJ 99, 1491, 1990 ) inciudes an investigation of the membership and variability of the variables, including those announced recently by Yao et al. (Acta Astron. Sin. 29, 243, 1988; ESO Mess. 50. 33, 1988). Kadia et al. (Sov. A. Lett. 14, 432, 1988; \& 15, 57, 1989) apply their method of determining reddening using Cacciaris photometry of the cluster RR Lyrae stars.

C1644-018 (NGC 6218, M12): The long-term behavior of V1 is discussed by Clement et al. (AJ 96, 1642. 1988).

C1659-262 (NGC 6273, M19): Galkina \& Samus (Astr. Tsirk. 1452, 3, 1986) discuss the long-term behavior of $\mathrm{V} 7$ and briefly discuss $\mathrm{V} 2$.

C1715+432 (NGC 6341, M92): Pilachowski (ApJ Lett. 326, L57. 1988) includes V45 in a paper on the abundance of oxygen in giant stars of the cluster.

C1716-184, (NGC 6333, M9): Values of $\triangle S$ are given by CS for V1 and V3.

C1732-447 (NGC 6388): Smith et al. (IAU Coll. 111, 285, 1989) report on CCD observations and announce at least 10 additional cluster RR Lyrae stars.

C1814-522 (NGC6584): Rodgers \& Harding (PASP 102, 235, 1990) give Ca abundances for V6, V10. V13, V28 and V37.

C1821-249 (NGC 6626, M28): Nook \& Cardelli (ApJ Lett. 346, L29, 1989) announce detection of an IR excess probably arising from a dust shell surrounding the RV Tauri star V17. A paper on carbon isotopic ratios in cluster giants by Smith \& Suntzeff (AJ 97, 1699. 1989) includes V8. Wehlau \& Clement (IBVS 3450, 1990) give a period for V7, a field Mira star. Two-color photographic photometry of the variables is presented by Wehlau \& Butterworth (AJ 1990, in press).

C1832-330 (NGC 6652): Hazen (AJ 97, 771, 1989) presents photometry for 24 new variables but finds no RR Lyrae stars which are likely to be cluster members.

C1850-087 (NGC 6712): An astrometric and photometric study by Cudworth (AJ 96, 105, 1988) includes an investigation of the cluster membership of the variables.

C1852-227 (NGC 6717): CS give $\triangle S$ values for the variable star SPZ2200.

C1908+009 (NGC 6760): Prosser (IBVS 3268, 1988) discusses V1 and V4 and includes a finding chart for the cluster variables.

C2050-127 (NGC 6981, M72): Rodgers \& Harding (PASP 102, 235, 1990) give Ca abundances for V3, $\mathrm{V} 10, \mathrm{~V} 23$ and $\mathrm{V} 25$.

C2127+119 (NGC 7078, M15): Kadla et al. (Izv. Glav. Obs. Pulkovo 205, 114, 1988) present identification charts and a catalogue for 123 variables. Yao (IBVS 3431, 1990) reports variability in the red giant K1040 = Sandage 6. Barlai (Comm. Konkoly Obs. 10, No.92, part 6, 1990) presents light curves for 55 variables based on almost 30 years of observations.

\section{POPULATION \| VARIABLES IN MAGELLANIC CLOUD CLUSTERS AND DWARF GALAXIES}

CCD photometry is presented for the RR Lyrae variables in the SMC cluster NGC 121 (Walker \& Mack, AJ 96, 872, 1988), and in the LMC clusters NGC 1786 (Walker \& Mack, AJ 96, 1362, 1988). NGC 1841 (Walker, AJ, in press) and NGC 2257 (Walker. AJ 98, 2086, 1989). Walker reports on his survey for 
variables in five SMC clusters (PASP 101, 570, 1989). Photometry of the distant cluster (or dwarf spheroidal galaxy) in Reticulum by Gratton \& Ortolani (AA 71, 131, 1987) includes the variables already known and 7 probable new variables. Nemec et al. (AJ 96, 528, 1988) report on a study of 95 variables in the Ursa Minor dwarf spheroidal galaxy and derive P-L relations for anomalous Cepheids corresponding to fundamental-mode and first-overtone pulsators. Saha \& Hoessel present finding charts. periods, and light curves for anomalous Cepheids and RR Lyrae stars in the dwarf elliptical galaxies NGC 147 (AJ 94, 1556, 1987; AJ 100, 108, 1990) and NGC 185 (AJ 99. 97, 1990). Also see the papers by Feast et al. and Frogel et al. cited in the section below on red variables.

\section{ANOMALOUS CEPHEIDS AND VARIABLE BLUE STRAGGLERS}

The number of known anomalous cepheids (AC) has increased during the past few years as more have been identified in Ursa Minor, NGC 147 and NGC 185, as mentioned above. A review of the subject by Nemec (IAU Coll 111, 215, 1989) discusses these variables and their relation to Population II blue stragglers which, like $A C$, have relatively large masses and appear in stellar systems of low density. $P-L$ relations for $A C$ pulsating in the fundamental-mode and the first-overtone are presented. A number of blue stragglers have been shown to be eclipsing binaries or periodic variables with periods from 44 min. to 5 hours, similar to SX Phe variables (Pop. II $\delta$ Scuti stars/Dwarf Cepheids) found in the galactic halo. See Da Costa (IAU Coll. 95, 579, 1987). Da Costa \& Norris (IAU Symp. 126, 681, 1988), Mateo et al. (AJ 100, 469. 1990), and Harris et al. (CCD's in Astronomy II. in press). Nemec and his collaborators have found more such variables in M71 and the Carina dwarf spheroidal galaxy.

\section{RED VARIABLES}

Feast et al. (MN 241, 375, 1989) use their PLC relation obtained from LMC Miras to estimate distances to galactic and $\mathrm{MC}$ clusters containing Mira stars. IR photometry of red variables in 13 galactic clusters is presented and discussed by Frogel \& Elias (ApJ 324, 823, 1988). Variability of AGB stars in MC clusters is discussed by Frogel et al. (ApJ 352,96, 1990). Studies which include red variables in a single cluster are already listed in the first section under the cluster name.

\section{PERIOD CHANGES, PULSATION MODELS AND FOURIER ANALYSIS OF LIGHT CURVES}

Lee (ApJ Lett., 1990, in press) compares period changes of RR Lyrae stars in 5 clusters to those predicted by synthetic $\mathrm{HB}$ models and concludes that the mean rate of change in each cluster depends of the cluster $\mathrm{HB}$ type and can be attributed to evolutionary effects. A less conventional method for producing period changes is suggested by Dearborn et al. (ApJ 354, 568, 1990) in their paper on HB stellar evolution including the effect of 'WIMPS'. A review by Cox (IAU Coll. 95, 161, 1987) discusses the theory for double-mode pulsation in RR Lyrae stars and the problems involved in using this pulsation to derive masses. Fokin (Nauchn. Inf. Vyp. 67. 98. 1989) describes a method of calculation of nonlinear stellar pulsations of $W$ Vir stars and presents the results from his model for M3 V155. Stellingwerf \& Dickens (ApJ 322, 133, 1987) give Fourier coefficients obtained for the RR Lyrae stars in NGC 6171 (C1629-129, M107). Simon (ApJ 328, 747, 1988) has shown that the $\phi_{12}$ Fourier phase parameter derived from light curves of a small sample of shorter-period RRab stars in $\omega$ Cen varies with metallicity in a way similar to that found for field RR Lyrae stars. Comparing the observations of $\omega$ Cen RRc and RRd stars to a large number of hydrodynamical pulsation models, Simon (ApJ Lett. 343. 17, 1989) investigates the accuracy of the physical data that may be derived by use of Fourier decomposition of these light curves. In particular, he finds a hint of a mass-He abundance relation for these stars.

\section{THE ABSOLUTE MAGNITUDES OF RR LYRAE STARS AND THE PERIOD-SHIFT EFFECT}

There is still disagreement among researchers as to origin of the Sandage period-shift effect and the slope of the relation between $\mathrm{HB}$ luminosity and metallicity. Discussions of these problems, and of their implications for determination of fundamental data such as distances and ages of clusters, can be found in papers by Tornambe (ESO Workshop 27. 307, 1987). Renzini \& Fusi Pecci (Ann. Rev. Astron. Astrophys. 26, 80, 1988) and Buonanno et al. (AA 216, 80, 1989). Longmore et al. (IAU Coll. 111, 273 \& 274, 1989) have used their IR P-L relation for RR Lyrae stars to derive cluster distances and find a strong correlation between period-shift and metallicity. Caputo et al. (AA Suppl. 68, 119, 1987) have compared observed properties of cluster RR Lyraes with those expected from synthetic HB's while Castellani \& Quarta (AA Suppl. 71, 1, 1987) and Caputo et al. have investigated the Oosterhoff dichotomy and confirmed the variation with metallicity of pulsational characteristics of ab-type variables (AA 222. 121. 1989). Papers on the $\omega$ Cen variables listed in the first section also discuss this question.

The range in absolute magnitude of RR Lyrae stars, both within a given cluster and from cluster to 
cluster, are discussed by Sandage (ApJ 350,603, ApJ 350,631, 1990), while various determinations of the slope of the absolute magnitude-metallicity relation are reviewed and compared by Sandage \& Cacciari (ApJ 350, 645. 1990) who confirm a higher value of the slope near 0.37. On the other hand. Lee et al. (ApJ 350, 155, 1990) use synthetic HB models to predict a slope of only 0.17 and find that the period-shift effect can be explained by changes in light-curve shape with metallicity and evolution off the $\mathrm{HB}$ in Oosterhoff type ll clusters.

\section{CATACLYSMIC VARIABLES AND PULSARS IN GLOBULAR CLUSTERS}

Observations during quiescence and outburst of V101, a CV in M5, are reported by Naylor et al. (MN 241, 25, 1989). Results indicate that V101 is probably a dwarf nova at the distance of M5. Shara et al. (AJ 99, 1858, 1990) have obtained the first outburst spectrum of M30 V4 as well as quiescence spectra of M5 V101 and Nova 1938 in M14, the first spectrum of a globular cluster nova. All three are confirmed as CV's but cluster membership, in particular for M30 V4, is in doubt.

The first confirmation of a pulsar in a globular cluster, that in M28, had just been announced when the last report in this series was written. Now, three years later, at least 18 have been reported (Romani, ApJ 357, 493, 1990; IAU Circs. 4974, 4988 \& 5013, 1990). Most of these have millisecond rotation periods and a few have been found to be in eclipsing binary systems. Kulkarni et al. (ApJ 356. 174. 1990) predict a galactic globular cluster population of about 10 000 pulsars. For a discussion of the considerable literature arising from these discoveries the reader is referred to the reports of Comm. 37 on Star Clusters, Comm. 40 on Radio Astronomy, and Comm. 42 on Close Binary Stars.

\section{Mira Variables and Related Objects}

\section{(M. W. Feast)}

A general review of pulsating red variables is given by Whitelock (in 'Confrontation between Stellar Pulsation and Evolution.' Bologna 1990). Other reviews can be found in 'From Mira Variables to Planetary Nebulae' (ed. Mennessier 1990), in IAU Symp. 148 (Wood) and the ESO-CTIO 1990 conference on galactic bulges (Feast \& Whitelock). A period-luminosity-temperature (infrared colour) relation has been found for LMC O-rich Miras (Feast et al. 1989. MN 241, 375). The explanation of this relation is either a spread of metallicities at a given period or decreasing envelope mass (Wood. IAU Symp. 148). Hughes and Wood (AJ 97, 1634: AJ 99, 784) have greatly increased the number of known LMC Miras. They find a major discrepancy between the observed number and predictions of AGB theory.

The $\mathrm{OH} / \mathrm{IR}$ variables (with some exceptions, e.g. supergiants) form an extension of the Mira sequence to longer periods. A large fraction of the objects discovered in the older $\mathrm{OH}$ surveys had periods greater than 1000 days and few had known optical counterparts. IRAS selected samples contain a much higher proportion of less massive, shorter period, stars overlapping in properties with the longest period optical Miras (cf. te Lintel Hekkert 1990. Thesis, Leiden), Whitelock et al. (MN 1990 and references there). These latter workers show that the new improved 'phase-lag' distances of $\mathrm{OH} / \mathrm{IR}$ stars by van Langevelde et al. (AA 1990) form a natural extension of the Mira PL relation to longer periods though the uncertainties in individual distances derived by this method are still large. They also note that data on LMC IRAS sources with periods greater than 1000 days (Whiteoak et al. IAU Symp. 148) fit an extrapolation of the Mira PL relation with little scatter and that their new IR observations of galactic bulge objects support a scenario in which the Mira phase is short lived and involves little change in stellar luminosity or log (period) but a large change in pulsation amplitude and in the amount of circumstellar dust. Contrary to earlier conclusions there are few, if any, Miras with $P \geq 1000$ days in the galactic bulge. placing more stringent limits on the ages and masses of bulge objects. Many new Mira variables have been detected in the region of the galactic bulge (Terzan \& Ounnas ESO preprint 611). In addition Haller (preprint) has found a large number of long period variables in the central $5 \times 5$ arcmins of the bulge.

Extensive searches for new $\mathrm{OH} / \mathrm{IR}$ sources have been made mainly on the basis of the IRAS catalogue (Sivagnanam et al. AA 233, 112; Eder et al. ApJ Suppl. 66, 183 and in press: Gaylard et al. MN 236. 247: Galt et al., AJ 98. 2182; te Lintel Hekkert 1990. Thesis; Whitelock et al. 1990. MN). Sivagnanam et al. (AA 206, 285; AA 211, 341) have surveyed known (optical) Miras for $\mathrm{OH}$ and discussed the periodshell expansion velocity relation. The reason why, at given IRAS colours, some stars show OH masers and some not has been much discussed (Lewis et al. AJ 94. 1025: Lawrence et al. AJ 99, 1232: Likkel ApJ 344,350 ) the cause may be due partly to the effect of binaries or lack of variability or asymmetric shells. However the main cause seems likely to be a previously unrecognized sensitivity limitation (Whitelock et al. 1990. MN). 
High resolution data shows the discrete nature of $\mathrm{OH}$ maser emission in $\mathrm{OH} / \mathrm{IR}$ stars (Zell \& Fix AJ 99, 314; cf. also Szymczak MN 237. 561). A model for the kinetic structure of the shell was constructed by Sun \& Kwok (AA 185, 2581). Sivagnanam et al. (AA 229, 171) studied OH hot spots in the envelope of the Mira $U$ Her and note the possible importance of $\mathrm{OH}$ emission objects as a possible link between the radio and optical reference frames. VLA observations lead to an axisymmetric. biconical model for the envelope structure of the Mira U Ori (Bowers \& Johnston ApJ 330, 339). Other OH/IR sources on which detailed studies of the shell structure have been carried out are the (probably bipolar) object $\mathrm{OH}$ 19.2-1.0 (Chapman MN 230, 415); $\mathrm{OH} 26.5+0.6$. OH 127.8-0.0 and IRC +10420 (speckle interferometry: Fix \& Cobb, ApJ 329, 290; VLA observations: Bowers \& Johnston, ApJ 354, 676). $\mathrm{OH}$ 127.8-0.0 may be a supergiant whilst a shell asymmetry for $\mathrm{OH} 26.5+0.6$ may be due to UV radiation from a nearby $O 8$ star. Smith \& Herman (AA 227, 147) give evidence for annealed silicates in the envelope of $\mathrm{OH} 138.0+7.3$ whilst Stencel et al. (ApJ 350, 245) have described a dust grain formation and annealing sequence. The possibility of distinguishing spectroscopically between $\mathrm{OH} / \mathrm{IR}$ Miras and supergiants was studied by Jones et al. (AJ 95. 158). For the OH/IR supergiants. polarimetry shows that there are significant interstellar and intrinsic components, implying both significant interstellar extinction and large shell asymmetries. Lewis (AJ 99, 710) has investigated the placing of circumstellar maser sources in an ordered sequence which may be a clue to their evolution. Bedijn (AA 205. 105) has discussed an evolutionary scenario in which Miras evolve into OH/IR sources with a large change in period (but see Whitelock et al. 1990). More, general, studies of masering in circumstellar shells have been carried out (Pichler \& Kegel, AA 214, 339; Rottgering AA 222, 125; Netzer \& Knapp ApJ 323, 734; Szymczak Astrophys. Space Sci. 141, 353).

Cesaroni et al. (AA Suppl. 76, 445) have published a catalogue of $\mathrm{H}_{2} \mathrm{O}$ masers north of -30 degrees and Comoretto et al. (AA Suppt. 84, 179), an atlas of 203 of these. Most sources are variable. Engel et al. (AA 191, 283) find $\mathrm{M}$-supergiant $\mathrm{H}_{2} \mathrm{O}$ masers are fairly stable, semiregular variables show strong variations and Miras and IRC sources somewhat less. They compare $\mathrm{H}_{2} \mathrm{O}$ velocity structure with $\mathrm{OH}$. Further $\mathrm{H}_{2} \mathrm{O}$ masers were detected and discussed by Deguchi et al. (MN 239, 825), including some with LRS spectra indicating (apparently incorrectly) SiC emission and by Lewis \& Engels (Nature 331. 49). Lindquist et al. (AA 229, 165) have found $\mathrm{H}_{2} \mathrm{O}$ masers in some $\mathrm{OH} / \mathrm{IR}$ stars close to the galactic centre. A correlation between the shape of the light curve and $\mathrm{H}_{2} \mathrm{O}$ masering or silicate dust emission was investigated by Vardya (AA 182, 75; AA 209, 165). Millimeter $\mathrm{H}_{2} \mathrm{O}$ emission was detected in VY $\mathrm{CMa}$ and $\mathrm{W}$ Hya and submillimeter $\mathrm{H}_{2} \mathrm{O}$ in VY CMa (Menter et al. ApJ 341, 291, ApJ 350, 241) and was explained in terms of collisional pumping (Neufeld \& Melnick ApJ 352. L9).

$\mathrm{SiO}$ masers are particularly interesting as coming from the inner region of the circumstellar shell only a few stellar radii from the stars surface. Polarization indicative of magnetic fields in the range $10-$ $100 \mathrm{G}$ was reported by Barvainis et al. (Nature 329, 613). Allen et al. (MN 326. 363: MN 243, 480) detected $\mathrm{SiO}$ masers in southern Miras. IRAS and symbiotic stars. The presence of SiO masering is strongly connected with large optical variability (presumably due to mass loss being pulsationally driven (see also Alcolea et al. AA 231, 431). They suggest the polarization seen in the SiO masers in R Aqr (a symbiotic star) is associated with magnetic fields. Gomez et al. (Rev. Mex 1990) also find new SiO (and $\mathrm{H}_{2} \mathrm{O}$ ) masers. The percentage of such objects decreases with [25-12] colour which they take to be an age indicator. Other surveys are: Alcolea et al. (AA 211. 187); Bujarrabal et al. (AA 175. 164), including evidence that the emission is radiatively rather than collisionally pumped. Struck-Marcell (ApJ 330, 986) suggests that SiO masers in Miras originate in the magnetospheres and orbital wakes of Jovian planets. A detailed study of the structure, polarization and variability of SiO masers in $\mathrm{A}$ Cas shows emission clumps distributed over a diameter of several stellar radii (Mclntosh et al. ApJ 337. 934). In another Mira, R Leo, and for the supergiants VY CMa and VX Sgr, high excitation SiO masers have been found (Jewell et al. ApJ 323, 749). The results seem to preclude simple theories of photon cascades within rotational ladders. For Miras the position of the SiO masers are within 0.35 arcsec of the optical position, but in the case of VY CMa, there is a displacement of 1.1 arcsec (Wright et al. AJ 99, 1299). Long term monitoring of SiO masers (Martinez et al. AA Suppl. 74, 273) shows a poor repeatability and the results suggest that the masers are formed very close to the stellar atmosphere. A study of $\mathrm{SiO}$ (and $\mathrm{CO}$ ) thermal emission from Miras leads to quite high mass loss rates of $\sim 10^{-6}$ solar masses per year (Bujarrabal et al. $\mathrm{AA}$ 219. 256). Observations of $\mathrm{CO}$ emission in O-rich and C-rich Miras and semiregulars suggests a distinct difference in outflow velocity between variables with periods greater or less than 500 days (Heske AA 229. 494). Engels and Heske (AA Suppl. 81, 323) compiled a reference catalogue (191 objects) of $\mathrm{SiO}$ maser and thermal emission.

The occurrence of silicate dust shells round a few carbon stars (many of them variable) may be an important clue in understanding late-AGB evolution. Lloyd Evans (MN 243, 336 and preprint) and 
Lambert et al. (AJ 99, 1612) confirm the Willems-de Jong hypothesis that all such $C$ stars are ${ }^{13} \mathrm{C}$ rich ( $J$ stars) and give evidence favouring the Lloyd Evans scenario of dust formed in a disc round a low mass companion from matter ejected by the primary before its atmosphere became carbon rich. No SiO masers or CO emission are found in these stars (Deguchi et al. AA 230. 339). HCN. CS and HNC have been detected in the circumstellar shells of some O-rich variables (Lindquist et al. AA 205. L15; Nercessian et al. AA 210, 225). departures from LTE seem necessary to explain these results. see also Sopka et al. (AA 210, 78). Some effects of the differing physical properties of carbon and silicate grains on the circumstellar shells of $C$ and $O$ rich Miras have been investigated by Lewis (AJ 98 , 1814 and preprint). $\mathrm{CO}$ observations of carbon variables show the presence of detached shells and indicates episodic mass loss, possibly connected with helium shell flashes (Olofsson et al. AA 230. L13). Le Bertre (AA 190, 79) discusses possible pulsation-related dust formation in the C-Mira R For. CO observations of carbon variables show a correlation between wind velocity and infrared excess (at moderate infrared excess only). CO observations show that the carbon variable $\mathrm{V} H y$ a is bipolar. The star has rapid rotation. possibly indicating spin up in a binary system (Kahane et al. ApJ 328, L25: Tsuji et al. ApJ 327. L23). Optical evidence of shock-excited emission lines from the (bipolar) jet of $V$ Hya has been obtained by Lloyd Evans (preprint). The high outflow velocities for the C stars RAFGL 2233 and 2901 probably require a binary model (Claussen \& Ziurys AA 231.73). The circumstellar envelope of o Ceti has been mapped in HI and CO (Bowers \& Knapp ApJ 332, 299; Planesas et al. ApJ 351. 263). In addition to a spherical envelope of mass $\sim 6 \times 10^{-4}$ solar masses there is evidence (from CO) of a bipolar flow which may be transitory. The oxygen Mira FY Aql is embedded in a reflection nebula (diameter 30 arcsec) (Hartmann \& Pogge ApJ 318. 363) and the oxygen Mira W Hya in a $\sim 30$ arcmin dust shell (temp $\sim 40 \mathrm{~K}$ ) (Hawkins AA 229, L5) is somewhat reminiscent of the shell round $R \mathrm{CrB}$.

Observational evidence indicates that mass loss rates are related to the amplitude of pulsation (e.g. Whitelock et al. MN 1990. see also Heske AA 208. 77 and the review by Morris PASP 99, 1115) but other processes may contribute e.g. radiation pressure from photons trapped in $\mathrm{H}_{2} \mathrm{O}$ rotation-vibration transitions (Elitzur et al. ApJ 341. L95) or acoustic waves (Pijper \& Habing AA 215, 334). Wave driven winds were also considered by Havnes et al. (AA 217, L13) and the minimum mass loss rate for dust driven winds by Gail \& Sedlmayr (AA 177, 186), whilst a detailed analysis of radiation pressure on circumstellar grains was made by Lefevre (AA 219, 265). Netzer (Ap' 342, 1068) showed that it is necessary to take into account infrared emission by dust to explain the velocity field in the dust shell of the supergiant VX Sgr. Detailed modelling of circumstellar dust emission has been carried out by Schutte \& Tielens (ApJ 343, 368; see also Fleisch et al. in 'Confrontation between Stellar Pulsation and Evolution,' 1990) whilst van der Veen \& Rugers (AA 226. 183) have compared dust. $C O$ and $O H$ mass loss rates. Le Bertre \& Schwarz (AA 229, 138) discuss the variable polarization in two IRAS Miras. Shock wave models for Miras and the resulting mass loss are discussed by Bowen (ApJ 329, 299) and Gillet (AA 190, 200; AA 192, 206) deduced shock velocities etc from high resolution profiles of $H(x$ in Miras. A shock model has also been derived from ultraviolet Mgll profiles in the Mira S Car (Bookbinder et al. ApJ 342, 516). The consequences of helium shell flashing for AGB evolution and for Miras in particular were discussed by Boothroyd \& Sackmann (ApJ 328, 632). The flashes could result in envelope instability and rapid mass loss at lower core masses than would otherwise be the case.

Effort continues to identify objects in the expected transition phase between Mira variables and planetary nebulae. These include discussions of the systematics of infrared (generally IRAS) colours as well as other data and their possible evolutionary significance (e.g. van der Veen et al. AA 226, 108; van der Veen AA 210, 727; Habing AA 194, 125; Kwok MN 244, 179; Garcia-Lario et al. AA Suppl. 82, 497. Lewis ApJ 338, 234), as well as infrared and radio studies (e.g. Kwok et al. ApJ 321, 975; Bowers \& Knapp ApJ 347, 325, Le Bertre AA 180, 160). IRAS 07027-7934 may be a link between OH/IR stars and carbon-rich planetary nebulae (Zijlstra et al. SAAO preprint 680). There remains the considerable problem of distinguishing between true transition objects and those which show peculiarities for other reasons such as binary interaction as in symbiotic stars (e.g. He 2-104, Schwarz et al. ApJ 344. L31; Lutz et al. PASP 101. 966). Bipolar optical/radio jet action in the symbiotic Mira R Aqr has been further studied (Hollis ApJ 351. L17; Kafatos ApJ 346, 991) and high resolution infrared spectroscopy (Hinkle et al. AJ 98. 1820) places some limits on the binary orbit. If the orbit is elliptical. Roche lobe overflow from the Mira may occur at periastron. High velocity emission lines suggesting bipolar flows have also been found in the semiregular symbiotic V1329 Cyg (Wallerstein et al. PASP 101. 189). A detailed study of the optical polarization in the bipolar nebula surrounding the 660 day variable $\mathrm{OH} 231.8+4.2$ shows amongst other things that the dust grains there are much smaller than those in the ISM (Scarrott et al. MN 243, 462). Monk et al. (MN 242, 457) now believe that the apparent extension of [Oll] emission in the symbiotic Mira RX Pup is a combination of atmospheric scattering and diffuse galactic emission. The 
symbiotic Mira V407 Cyg has a very long period (745 d) but a relatively thin dust shell thus offering the opportunity to study the central Mira (single Miras of this period are generally heavily enshrouded by dust) (Munari et al. MN 242, 653). Other symbiotics discussed include. BF Cyg (Mikolajewska et al. AJ 98, 1427; Fernandez-Castro et al. AA 227, 422; Isliker et al. AA 219, 271), HM Sge (Leahy et al. AJ 99 , 1926); V352 Aql and V840 Cen (Duerbeck \& Seitter PASP 101, 673) with more general discussions by Seaquist \& Taylor (ApJ 349. 313, radio properties). Formiggini \& Leibowitz (AA 227, 121, light curves) and Schild (MN 240,63) who discusses AGB evolution on the hypotheses of Mira evolution from short to long period (but cf. Whitelock et al. MN 1990).

Cannizzo et al. (ApJ 357, 235) find no evidence for deterministic chaos in the light curves of three Miras. The variations are basically periodic although in one of these (R Leo) Hoeppe (AA 178, 131) found evidence in data from 1757 to 1984 of both sudden irregular changes and long term variations in minimum brightness. On the other hand the irregular variability of supergiants may be an example of chaotic behaviour (Buchler \& Goupil AA 190, 137). The multimode behaviour of the semiregular Z Sge was discussed by Mantegazza (AA 196, 109). New periods have been determined for a number of southern Miras (Shawl \& Bord AJ 93, 898; AJ 99, 917). Other work includes possible period changes in the Mira R Aur (Lloyd Obs 109, 146), periodicities in the semiregular EU Del (Percy et al. PASP 101, 893) and classification of Mira light curve shapes (Vardya. AA Suppl. 73, 181). New absorption line radial velocities for 20 Miras (Barbier et al. AA Suppl. 72,463 ) indicate an infall of matter relative to the mean $\mathrm{OH}$ velocity which must be accounted for in any dynamic model. In semiregular variabies with $\mathrm{H}_{2} \mathrm{O}$ masers a difference between the maser and optical velocity is due to the expansion of the masering region with only the near side masers seen (Wallerstein \& Dominy ApJ 326, 292).

Bessell et al. (AA 213, 209) have constructed model photospheres for oxygen Miras which reproduce, at least approximately, infrared colours and some molecular band strengths; they also discuss the Mira temperature scale. The temperature scale for Miras depends on angular diameters determined from lunar occultations or from speckle interferometry and depends on the adopted limb darkening coefficients. Beach et al. (ApJ 329, 241) show that the limb darkening can be significantly affected by pulsation effects in the stellar atmosphere thus affecting the temperature scale (see also Scholz \& Takeda AA 186, 200). Extensive spectral classification of 72 southern Miras through their cycles leads Crowe \& Garrison (ApJ Suppl. 66, 69) to a two shock model. Hron (in 'Confrontation between Stellar Pulsation and Evolution' 1990) is carrying out a survey of short period Miras in JHKL.

C,N,O abundances in carbon stars, including Miras, were determined by Lambert et al. (ApJ Suppl. 62. 373) whilst Little et al. (AJ 94, 981) surveyed 279 late type stars for technetium. The presence of Tc correlates strongly with light variability; amongst $M$ types it is restricted to the Miras. Lattanzio \& Malaney (ApJ 347, 989) explain this result by photofission production of Tc (rather than s-process production). S-type stars (non-Miras) without Tc are interpreted as the result of binary interaction (Brown et al. AJ 99, 1930). Jorgensen (ApJ 344, 901; see also AA 232, 420) has computed a synthetic spectrum which reproduces the intrared spectrum of the carbon star TX PsC. The parameters of M type Miras have been derived from model fits to IRAS LRS spectra (Onaka et al. AA 218. 169; AA Suppl. 81, 261). The results lead to a discussion of dust in the circumstellar shell which is modelled with a mixture of amorphous magnesium silicate and aluminium oxide. In oxygen rich circumstellar envelopes photochemical calculates predict significant amounts of $\mathrm{H}_{3} \mathrm{O}+$ and $\mathrm{HCO}+$ (Mamon et al. ApJ 323, 306). The circumstellar chemistry of the carbon Mira IRC +10216 (CW Leo) continues to occupy attention. Work includes the detection of CP (Gu'lin et al. AA 230. L9) and $\mathrm{C}_{4}$ Si (Ohishi et al. ApJ 345. L83), the distribution of SiS in the envelope (Beiging \& Nguyen-Quang-Rieu ApJ 343, L25), the discovery of cm continuum (as well as in CRL 2688) (Sahai et al. AA 220. 92). Howe \& Millar (MN 244, 444) discuss the formation of carbon chain molecules in IRC +10216 and Orofino et al. (AA 231, 105) model the dust emission on the basis of laboratory measurements of the properties of amorphous carbon grains. LP And (Alksnis IBVS 3396), a 614 day carbon Mira with a thick dust shell, may be similar to IRC +10214 . The shell of IRC +10216 (as well as that of some other Miras) has been resolved at $10 \mu \mathrm{m}$ using speckle interferometry (Benson et al. AJ 97. 1763). Herman (AA Suppl. 74, 133) selects AGB variables from the IRAS catalogue and concludes amongst other things that the ratio of $C$-rich to O-rich stars is 1 to 5 (twice as high as previously thought).

Besides work in our own Galaxy and the Magellanic Cloud work on long period variables has been carried out in two other galaxies. Infrared photometry of (mainly) supergiant red variables in M33 has been used to derive a distance for this galaxy of $760 \mathrm{kpc}$ (Mould et al. AJ 349, 503) and 30 long period variables (all of relatively low amplitude) have been found in the Fornax dwarf spheroidal (Demers \& Irwin MN 226. 943); about $25 \%$ of them appear to be carbon stars. 


\section{Asteroseismology of Compact Pulsators}

\section{(D. E. Winget)}

There has been considerable quantitative and qualitative progress in the study of the compact pulsators since the last summaries in these pages (Winget, Trans. IAU, vol. XXB, 278, 1988; Cox. Trans. IAU, vol. XXB, 1988). This review will be restricted to only a few of the most important developments since those earlier reviews. For more detailed and comprehensive recent reviews of this field, see Kawaler \& Hansen ('White Dwarfs', in IAU Coll. 114, 97, 1989), \& Kawaler (in 'Confrontation Between Stellar Pulsation and Evolution,' Bologna. in press).

The compact pulsators occupy three widely separated regions of the $\mathrm{H}-\mathrm{R}$ diagram which span an enormous temperature range from the pulsating planetary nebula nuclei (PNNV stars) and the hot prewhite dwarf stars (DOV stars), both in excess of $100000 \mathrm{~K}$. through the pulsating DB white dwarfs (DBV stars) near $25000 \mathrm{~K}$, all the the way down to the ZZ Ceti stars (DAV stars) near $12000 \mathrm{~K}$.

All of the compact pulsators have multi-periodic low-amplitude light variations. Pulsation has been detected with periods ranging from roughly seventy seconds to several thousand seconds with amplitudes of individual periods typically much less than $1 \%$; the pulsations are thought to be the result of self-excited nonradial g-modes.

The number of pulsations observed in individual pulsators is typically greater than that for any non-solar pulsators (Unno et al., 'Nonradial Oscillations of Stars.' 1989). This, coupled with the result that the pulsators are otherwise normal white dwarf or pre-white dwarf stars. makes the compact pulsators particularly ripe for asteroseismological investigations.

Recently, significant progress has been made in defining the temperature location of both the hottest, DOV, and the coolest. DAV, regions of instability. The high temperatures and luminosities of the DOV stars have made the determination of their temperatures extremely difficult from both a theoretical and an observational perspective. Work by Barstow \& Holberg (MN in press) using EXOSAT data and LTE model atmospheres, and work by Werner et al. (in 'Proc. Hot Star Workshop: Intrinsic Properties of Hot Luminous Stars.' Bouider, USA, in press) employing NLTE model atmospheres combined with IUE and new optical data. have helped to better constrain the temperatures and gravities. Both sets of investigators find temperatures well in excess of $100000 \mathrm{~K}$ and $\log \mathrm{g}$ of about 7 for all the DOV stars.

Differences in the results of the two groups, however, remain significant. For example, in the case of the proto-type DOV. PG 1159-035 (GW Vir. McGraw's star). Barstow \& Holberg find T = $123500 \mathrm{~K}$. while Werner et al. find $T=140000 \mathrm{~K}$. More important perhaps are discrepancies in the CNO:He ratios. Werner et al. find significantly larger values than Barstow \& Holberg. Accurate determinations of the values of this ratio in the DOV and spectroscopically similar objects are crucial if we are to understand the driving in these stars. Currently, the best model is that of cyclic ionization of $\mathrm{C}$ and $\mathrm{O}$ as demonstrated by Starrfield et al. (ApJ 293, L23. 1989, see also Cox et al. in 'Confrontation Between Stellar Pulsation and Evolution,' Bologna, in press); they find that this driving is quite sensitive to the $\mathrm{CNO}: \mathrm{He}$ ratio. At the present, the values found by Werner et al. are consistent with this driving mechanism, while those of Barstow \& Holberg are not.

This kind of driving places a difficult constraint on the evolution of the DOV stars. A helium layer thin enough to be above the driving region will probably mix shortly after the He becomes convective down to the transition zone boundary (as the star cools below roughiy $25000 \mathrm{~K}$ ). Thus the descendants of the DOV stars would have very little, if any. $\mathrm{H}$ or $\mathrm{He}$ at the photosphere. The high space density of the DOV stars suggests that their descendants are a large fraction of the total white dwarf population, and we simply do not observe enough objects with this sort of surface compostion. Vauclair (Proc. Oji Internat. Seminar. eds. Osaki \& Shibahashi, 1990) has suggested a resolution to this difficulty. In this model it is still CNO-partial ionization that drives the pulsations, however, the CNO is enhanced in the subsurface driving region by chemical diffusion.

The importance of the determination of the abundances in the DOV stars is further underscored by our current lack of an explanation of why apparently spectroscopically identical objects (the spectroscopic class of PG 1159-035 stars) are roughly evenly divided between pulsators and nonpulsators. It may be that this is just the result of 'He-poisoning' of the driving region: Starrfield (2nd Conference on Faint Blue Stars. Tucson, 309. 1987) has shown that if the He abundance is greater than $30 \%$ in the driving region, the star will not be pulsationally unstable. Another possibility is that there are significant surface temperature differences between the pulsators and nonpulsators.

Clearly, we need better determinations of photospheric temperatures and abundances in the near future. and this work should receive a high priority. 
Considerable work has also been done recently to refine our understanding of the location of the DAV instability strip. This work has incorporated extensive IUE observations (Lamontagne et al. 1989, IAU Coll. 114, 240) as well as new. higher signal-to-noise optical spectroscopy coupled with new model atmosphere calculations (Daou et al. ApJ, in press). The best value from these works seems to be from roughly $13000 \mathrm{~K}$ for the blue edge to $11500 \mathrm{~K}$ for the red edge. The above work is particularly important in the context of the determination of the ratio of nonvariables to variables within the strip; it underscores the problem of using inhomogeneous sets of photometric colors -- even narrowband colors. Indeed. Bergeron \& McGraw (ApJ Letters 352, L45, 1990) used high-resolution, high signal-to-noise data to demonstrate that the star GD 165 lay within the strip (as determined in the same way). Their highspeed photometric observations subsequently demonstrated that the object was indeed a pulsating variable star, and the 21st known DAV. This powerfully illustrates the importance of accurately determining the temperatures of DA stars in and around the instability strip in determining the ratio of variables to nonvariables within the strip. No DAV star with accurately determined relative temperatures placing it inside the strip has yet been found to be constant

The census of other compact pulsators has increased recently. Lo-4 became the 2nd known pulsating planetary nebula nucleus (Bond \& Ciardullo 1989. IAU Coll. 114, 473). In addition two new DBV stars have been added to the fold, bringing the total known to seven. The two stars are KUV 05134+2605 (Grauer et al. 1989. AJ 98, 2221) and CBS 114 (Winget \& Claver 1989. IAU Coll. 114. 290). On the basis of limited photometric observations, both these objects appear to be typical complex DBV stars: PG $1351+489$ remains unique among the DBV stars in its simplicity.

On the theoretical front, theorists have been busy constructing new tools for the study of pulsating compact stars. Tassoul et al. (ApJ Suppl. 72. 335, 1990) have published an extensive grid of evolutionary models, as have Wood (Ph.D. Thesis, Univ. Texas, 1990) and Wood et al. (preprint).

These equilibrium models are being pushed to the limit by new equation solvers. The first of these solves the equations of nonradial pulsation in a fully Lagrangian form (Pesnell 1990, preprint; Cox et al 1987. ApJ 317, 303). Although this technique is promising, particularly for non-degenerate stars, the current equilibrium models of compact stars are not currently up to the task. The Brunt-Vaisala frequency is so low in the deep interior of the models that the zone-to-zone differencing inherent in this technique is too noisy for this implementaion. The problems arising from this have been well documented (Brassard et al. 1989, IAU Coll. 114, 263; Brassard et al., ApJ, in press).

The second technique uses the finite element method and leads to marked improvements in adiabatic period determinations (Brassard et al., in 'Confrontation Between Stellar Pulsation and Evolution, Bologna, in press). This method is very useful -- even with the current noisy generation of models -- for computation of accurate periods and eigenfunctions needed for computation of rates of period change. mode trapping and period spacing.

A number of theoretical investigations -- built on the extensive unpublished work of C.J. Hansen -demonstrate that mode-trapping not only helps with the mode selection problem in DAV stars. but can be used to measure the hydrogen surface-layer mass in many DAV stars icf. Brassard et al. 1989, IAU Coll. 114, 258; Bradley \& Winget, ApJ, in press; Kawaler \& Weiss, Oji Int. Seminar, eds. Osaki \&Shibahashi, in press). In addition, Kawaler (in 'Confrontations Between Stellar Pulsation and Evolution,' Bologna, in press) shows that the previously mysterious minus sign in the observed $\mathrm{dP} / \mathrm{dt}$ for the $516 \mathrm{~s}$ mode of the DOV star PG 1159-035 is easily understood if the mode is trapped in the outer envelope. Then the contraction in the outer envelope dominates the cooling in the core, resuiting in a negative $\mathrm{dP} / \mathrm{dt}$ as observed.

The introduction of a coordinated global network for high-speed photometry, dubbed the Whole Earth Telescope (hereafter WET), has produced a revolution in the field of compact pulsating white dwarfs. The purpose of these observations is twofold: first, to provide continuous (gap-free) data of sufficient time-base to resolve the complex light curves of the compact pulsators, and second to provide the kind of data density necessary to produce accurate pulse phase measurements. as well as detect low amplitude modes. This project has been described in detail by Nather et al. (ApJ, in press). The results to date have exceeded the most optimistic expectations, and I can only begin to scratch the surface of them here.

Observations of the complex DAV G29-38 have revealed large phase variations (more than a third of a cycle) in one mode with no corresponding amplitude changes (Winget et al. 1990, ApJ 357 , 630). This is the first unambiguous demonstration of real instability in the light curve (i.e. not due to beating of closely spaced, but stable, modes).

Due primarily to the increased data density from WET, G117-B15A has finally surrended a value of $\mathrm{dP} / \mathrm{dt}$ for its dominant mode: $1.2 \pm 0.4 \times 10^{-14}$ (Kepler et al., preprint). This value is large enough to provide 
a real challenge for conventional evolution theory.

The gap-free data from a WET run has enabled Winget et al. (preprint) to completely resolve the light curve of PG 1159-035 in a wide range of frequency. They detected 125 individual frequencies of which 101 give specific quantized pulsation modes. The agreement with existing asymptotic theory has been spectacular. On the basis of this they determine the total stellar mass ( 0.59 solar masses), the rotation timescale $(1.38 \mathrm{~d})$, and constrain the magnetic field (B , $6000 \mathrm{G}$ ). All of the identified modes are consistent with either $\ell=1$ or 2 , and the identification of twenty consecutive radial overtones of $\ell=1$ allows the first direct detailed comparison of period spacing with theoretical models. The mode-trapping revealed in this comparison implies that the star is compositionally stratified.

Note that this last result presents a dilemma. If we see mode-trapping, the star is compositionally stratified in the region of period formation. If the driving is due to $\mathrm{C} / \mathrm{O}$ partial ionization, then we must have significant $\mathrm{C} / \mathrm{O}$ in the adiabatic/nonadiabatic transition zone where the driving occurs. The adiabatic/nonadiabatic transition zone is well outside (higher in the envelope) the region of period formation. so the implication is that the composition transition must be to a core of material significantly heavier than $\mathrm{C} / \mathrm{O}$. How the star may have produced such a core is difficult to understand theoretically at the present, but then nature never seems to be discouraged by our theoretical difficulties. A way out of this dilemma is provided by Vauclair's (see above) model for the driving. This may allow a cloud of $\mathrm{C} / \mathrm{O}$ to be supported in the driving region itself, and still make the transition from He to $\mathrm{C} / \mathrm{O}$ deeper in the star.

During the May 1990 WET run the value of using large telescopes was also underscored. Using the CFHT during the run Fontaine. Vauclair \& Bergeron found at least 15 bands of power in an individual run of less than 4 hours on the DOV star PG $1707+427$. Based on previous observations on telescopes with apertures of approximately 2 m or less, Grauer et al. (IAU Coll. 114, 119, 1989) had identified only two bands of power. The new results contain enough information to determine a preliminary mass using the methods of Kawaler (IAU Coll. 95. 297. 1987).

When this powerful new tool is applied to increasing numbers of pulsating compact stars, our understanding of their structure and evolution will be revolutionized.

\section{Theory of Stellar Pulsation}

(S. M. Morgan and A. N. Cox)

\section{INTRODUCTION}

This review is not complete in several respects because of space limitations. There are essentially no solar oscillation references, and the white dwarf research is reported only minimally.

There have been several international conferences where papers on stellar pulsation theory were presented. These include:

- 'Multimode Stellar Pulsations.' Budapest. Hungary. Sept. 1987 (ed. Kovacs et al., Konkoly Obs. and Kultura, 1988, in the following referred to as MUL),

- 'The Use of Pulsating Stars in Fundamental Problems of Astronomy.' IAU Coll. 111 (ed. Schmidt. Cambridge University Press, 1989, in the following referred to as USE),

- 'Seismology of the Sun and Sun-like Stars,' Tenerife, Spain, Sept. 1988, (ed. Rolfe, ESA Publ. Div., 1988).

- 'Inside the Sun,' IAU Coll. 121, Versailles. France, May 1989, (eds. Berthomieu \& Cribier, Kluwer, 1990).

- 'White Dwarts,' IAU Coll. 114. Dartmouth College. August 14-19, 1988 (ed. Wegner. Springer Verlag. 1989), and

- 'Confrontation Between Stellar Pulsation and Evolution;' Bologna Italy, May 1990. The Bologna meeting is too recent for inclusion in this report.

Other conference proceedings that were published during this time include

'Advances in Helio- and Asteroseismology,' IAU Symposium 123, Aarhus. Denmark. 1986 leds. Christensen-Dalsgaard \& Frandsen. Reidel, 1988),

- 'Stellar Puisation' - A Memorial to John P. Cox. Los Alamos, New Mexico, August, 1986 leds. Cox et al.. Springer-Verlag. 1987, in the following referred to as STE).

\section{CHAOS AND MODE RESONANCES}

Chaotic behavior in so-called regular variable stars has been extensively studied during this time. A review of such behavior is given by Buchler ('Chaotic Phenomena in Astrophysics,' eds. Buchler \& 
Eichhorn. N. Y. Academy of Sciences. 1987, p. 37). A review of multimode behavior in Cepheids and other supergiants is given by Szabados (MUL p. 1). Aikawa \& Bruegman (Astrophys. Space Sci. 137. 115) examined the pulsations of yellow supergiants under subharmonic resonances and found that generation of subharmonics may be effective in leading to chaotic oscillations in deterministic systems. Aikawa (Astrophys. Space Sci. 149. 149) studied a model which has a stable limit cycle but was very close to the transition from limit cycles to type I intermittency. From the work integral for nonlinear oscillations with various amplitudes, it was shown that the model has a stable limit cycle and also an unstable fixed point beyond the limit cycle. Aikawa \& Whitney (ApJ 328, 187) examined oscillations of a two-shell model of a star undergoing nonlinear conservative pulsations and compared it with those of a low-order approximation of a fully nonlinear model. They find that low-order approximations artificially induce strong nonlinear effects and are not valid indicators of the onset of chaos in conservative models with few degrees of freedom. Buchler \& Goupil (AA 190, 137) investigated the possibility that some irregular variability observed in supergiant variables has its origin in an underlying chaotic attracter. Kovacs et al. (ApJ 319. 327) use time dependent Fourier analysis to study the variation of the modal content of the numerical hydrodynamic integration of nonlinear RR Lyrae models.

The importance of the 2:1 resonance between the fundamental and overtone periods in the Hertzsprung progression of the bump cepheids is determined by Kovacs \& Buchler (ApJ 346. 898). Analysis of radial velocity data of 57 type I Galactic Cepheids appears to confirm this (Kovacs et al. ApJ 351. 606). Period doubling in Cepheid models is observed as the effective temperature is changed in models produced by Moskalik \& Buchler (ApJ 355, 590). They find in the weakly dissipative Pop. I Cepheids a single period doubling and subsequent undoubling. while for the strongly dissipative population II Cepheids a cascade of period doublings and chaos can occur. The dominance of 2:1 resonance between the fundamental and the second overtone is established in a sequence of radiative Cepheid models by Buchler et al. (ApJ 351, 617). In other models, the effects of a 3:1 resonance is studied and compared to those of 2:1 resonances. In some cases it may not be easy to discriminate in observational data between these two types of coupling (Moskalik \& Buchler. ApJ 341. 997). The stability of a three-mode limit cycle against the decay into a pair of damped modes is studied by Moskalik (MUL p. 149). Resonant three-mode coupling in beat Cepheids is examined by Kovacs \& Kollath (MUL p. 33). Two and three mode resonances involving the fundamental and the first six radial overtones are investigated by Petersen (AA 226. 151).

Nonlinearly pulsating models of RR Lyraes with purely radiative envelopes are computed to study the modal selection properties. With different masses, different pulsational properties such as bifurcations, resonances and non resonant double mode pulsations are seen (Kovacs \& Buchler. ApJ 324. 1026). The amplitude equation formalism and its success in describing the nonlinear behavior of classical pulsators is reviewed by Buchler (MUL p. 71). Nonresonant three mode coupling is examined by Verheest (Astrophys. Space Sci. 166, 77) in models with two linearly unstable modes in the presence of a representative stable or slave mode. One model results in a double mode pulsator, while other models evolve towards classic pulsators. Equations with nonlinear and nonadiabatic terms are used to describe a simple oscillator which shows period doubling and results in chaotic oscillations (Tanaka \& Takeuti, Astrophys. Space Sci. 148, 229). Nonlinear effects can perhaps result in chaotic motions leading to irregular stellar variability. Chaotic behavior in stars such as W Vir. RV Tau and yellow semiregular variables were modeled by Buchler et al. (MUL p. 265). Kovacs \& Buchler (ApJ 334, 971). Aikawa (Astrophys. Space Sci. 164, 295), Foken (USE p. 265) and Glasner \& Buchler (USE p. 267).

\section{INPUT PHYSICS}

The various theories of time dependent convection were reviewed by Baker (in 'Physical Processes in Comets. Stars and Active Galaxies,' ed. Hillebrandt et al., Springer Verlag. 1987, p. 105), along with its application to pulsating stars. Ostlie \& Cox (STE p. 243) model nonlinear RR Lyrae stars with time dependent convection and find that the pulsation decreases the convective flux. while the maximum flux occurs during the compression phase which limits the final amplitude. Cox \& Starrfield (USE p. 259) use linear theory time dependent convection on models of DA and DB white dwarfs and find that radial pulsations are stabilized in the models in agreement with observations. Ostlie (USE $p .281$ ) uses nonlinear models with time dependent convection to study its effect on the growth rates of doublemode RR Lyrae stars. The fundamental mode growth rate is decreased, while the first overtone is increased. In models with enhanced opacity (see below), ihe linear growth rates for both modes are lowered, while the nonlinear growth rates increase.

Changing the opacity of RR Lyrae and Classical Cepheids to remove period discrepancies has been promoted for some time. The effects of altering the opacities of such objects were investigated by 
Andreasen (AA 201, 72) and Andreasen \& Petersen (AA 192, L4) resulting in the apparent removal of the period discrepancy for double mode Cepheids and $\delta$ Scuti stars with ad hoc opacity enhancements. An examination of the contribution of iron lines to opacities by Rozsnyai (ApJ 341,414) resulted in opacities similar to those used by Andreasen. Mihalas (USE p. 59) reports that recalculations of opacities and equations of state are underway.

Weight functions in adiabatic stellar pulsations were studied by Pesnell (PASP 99. 975) as well as their variation with the adiabatic index $\gamma$. Nonradial oscillations of a star with variations of the ratio of specific heats were studied by Batia (Astrophys. Space Sci. 166. 219).

\section{METHODS OF MODELING STELLAR PULSATION}

In 'Nonradial Oscillations of Stars' (Unno et al., Univ. Tokyo Press, 1989), observations of nonradial oscillations, methods of calculating adiabatic and nonadiabatic oscillations, the effects of rotation and applications of theory are discussed. Davis (MUL p. 87) reviews the development of nonlinear stellar pulsation codes. Ipser \& Lindblom (ApJ 355, 226) construct rapidly rotating inhomogeneous Newtonian models and derive numerical methods for solving the eigenvalue problem for such a system. Methods of extracting eigenfrequencies of high order radial $\mathbf{p}$-modes with a precision of one part in 500000 and the requirements for the input stellar model are given by Mullan \& Ulrich (ApJ 331, 1013). According to Takeuti (Astrophys. Space Sci. 136, 129) the transitions from regular pulsations to irregular pulsations can be explained by the dependence of damping during an oscillation on the preceding amplitude. The variation of pulsation of a star evolving on its Helmholtz-Kelvin time scale is investigated by Bruggen \& Smeyers (AA 186. 170). A decrease or increase of the isentropic sound velocity in a mass layer contributes to an increase or a decrease of the period.

The modes of oscillation for 15 solar-mass rigidly rotating stellar models are studied by Clement (ApJ 339, 1022). It is found that except for very fast rotation, the $p$ - and $g$-mode periods can be computed accurately without the toroidal basis vectors. Higher order asymptotic approximations for $p-$ and g-modes of radial orders of a spherically symmetric star are developed by Smeyers \& Tassoul (ApJ Suppl. 65. 429).

\section{MODELING OF MASSIVE PULSATING STARS}

B Star pulsation theory is reviewed by Cox (STE p. 36) along with a discussion of possible pulsation mechanisms. Be star pulsations are reviewed by Baade ('Physics of Be Stars.' ed. Slettebak \& Snow, Cambridge University Press, 1987). including the current problems in Be pulsation theory.

Lee \& Saio (STE p. 102) model uniformly rotating stars and find that overstable convective (inertial) modes could penetrate into the envelopes of $B$ stars as a result of their resonance coupling with high order g-modes in the envelope. Massive rotating star stability is examined by Narasimha \& Sreenivasan (STE p. 106) where they find all radial modes stabte as well as p-modes and g-modes with $\ell$ between 4 and 10 .

The upper ZAMS mass limit for pulsating stars is examined by Odell et al. (STE p. 47) and Cahn et al. (STE $p$. 51). The former find the maximum mass for stars that are unstabie in the radial fundamental mode to be about 100 solar masses. while the overtones are stable. Nonradial modes in the same models are all stable. The latter use an improved linear nonadiabatic code and find the upper mass limit for pulsation to be 80 solar masses.

\section{MODELING OF LONG PERIOD VARIABLES (Miras, $\mathrm{R}$ CrB)}

A review of long period variables is given by Wood (STE p. 250), including observations, pulsation modes. mass loss and causes of mass loss. The dynamical structure and behavior of Mira-like stars is examined by Bowen (ApJ 329, 299). Linear nonadiabatic radial pulsation models of $\mathrm{R}$ CrB stars are examined by Weiss (AA 185, 178) who finds most models have at least one unstable mode. Periods and the blue edge of the instability strip agree well with observations. Saio \& Jeffrey (ApJ 328. 714) find for luminous hot helium stars that there exist unstable pulsation modes even at $T_{\text {eff }} \sim 30000 \mathrm{~K}$. Saio (MN 235.203 ) models helium stars to find mass-luminosity relations and determines that hotter puisating stars would have faster period changes. Fadeyev (MN 244. 225) made hydrodynamic calculations of radial nonlinear oscillations of helium stars and finds for models with 8000 ' $T_{\text {eff }}$ ' $30000 \mathrm{~K}$ and bolometric luminosities of $2.03 \times 10^{4}$ solar luminosities to be unstable, with the light amplitude decreasing with increasing $\mathrm{T}_{\text {eff }}$. Proffitt \& Cox (STE p. 379) study the pulsations of R CrB stars with a nonlinear hydrodynamic code and find a double periodicity. 


\section{MODELING OF PULSATING Ap AND $\delta$ SCUTI STARS}

A review of rapidly oscillating Ap stars and $\delta$ Scuti Stars is given by Shibahashi (STE p. 112). The various excitation mechanisms for Ap Stars and their relation to $\delta$ Scuti stars are discussed. Matthews (MN 235, 7P) suggests that the ionization of SilV in the envelopes of Ap stars could be a source of pulsational driving. The effects of combining a weak, axisymmetric magnetic field and slow rotation on the adiabatic pulsation frequencies of Ap stars are studied by Pesnell (ApJ 339. 1038). Standing wave patterns on the surface are produced when the magnetic field dominates, while in the case of the rotation dominating, the standing waves are split into the usual running waves. Antonello and Poretti (USE p. 249) find that unusual light curves of $\delta$ Scuti stars could be related to the poor helium content of the atmospheres when compared to models. Nonlinear effects in $\delta$ Scuti and ZZ Ceti stars are discussed by Dziembowski (MUL p. 127). The effects of rotation on the amplitudes of $\delta$ Scuti stars are studied by Krolikowski \& Dziembowski (MUL p. 141) and Dziembowski et a!. (Acta Astron. 38 , 61) who find that amplitudes are significantly lowered for $V_{\text {rot }} \cdot 20 \mathrm{~km} / \mathrm{s}$.

\section{MODELING OF CLASSICAL PULSATORS (Cepheids, RR Lyrae stars)}

Hydrodynamic modeling of Cepheids was done by Uji-lye et al. (STE p. 204) and Aikawa (STE p. 208) to look for modal coupling and to reproduce bump Cepheid features. Cox \& Proffitt (ApJ 324. 1042) studied anomalous Cepheids and conclude that such objects are formed from merged binaries. Carson \& Stothers (ApJ 328. 196) determine that if the luminosities of bump Cepheids were to be uniformly increased by 0.5 magnitudes, then these objects will have pulsation masses similar to their evolutionary masses. The effects of mass loss on Cepheid pulsations were examined by Li \& Huang (AA 229, 469), with no noticeable effect on the location of the instability strip or the period-radius relation.

Stothers (ApJ 319. 260) finds the distribution of periods of RR Lyrae stars in globular clusters can be accounted for adequately by fundamental and first overtone pulsators, while there does not appear to be any second overtone pulsators.

\section{MODELING OF WHITE DWARFS AND CENTRAL STARS OF PLANETARY NEBULAE}

Starrfield (STE p. 332) reviews the various types of pulsating stars that are produced during the planetary nebula stage, including ZZ Ceti. DBV stars, and GW Vir stars. Winget reviews multimode white dwarf pulsators including DOV. PNNV, DBV and ZZ Ceti Stars (MUL p. 181). Pesnell (ApJ 314. 598) discusses a new driving mechanism termed 'convective blocking' for model white dwarf stars. which is analogous to the $\kappa$ and $\gamma$ mechanisms in Cepheids. The pulsations of white dwarfs with a thick hydrogen shell are examined by Cox et al. (STE p. 358) and they find that radial pulsations should be produced. The effects of compositional stratification on the evolution of the oscillation spectrum of models evolving through the ZZ Ceti instability strip is studied by Winget (MUL p. 199).

Warner (STE p. 384) reviews the status of pulsations in cataclysmic variables such as DQ Her. Dwarf nova. QPOs, x-ray oscillators, and white dwarfs. Evolutionary models of white dwarfs that would be useful for pulsation studies were produced by Tassoul et al. (ApJ Suppl. 72, 335). Musielak \& Fontenla (ApJ 346. 435) examine the amplitudes of p-mode oscillations corresponding to trapped acoustic waves with small angular wave numbers. Das et al. (ApJ 346, 289) investigate the occurrence of multimode pulsations in degenerate stellar models. The occurrence of quasi-periodic motions is possible in these models. Kawaler (ApJ 334. 220) studies the occurrence of short period gmode pulsations for models of planetary nebula nuclei, and finds that such modes are driven by the $\epsilon$ mechanism at the location of nuclear burning shells, and the absence of pulsations in such objects could be due to an insufficient amount of hydrogen.

\section{MODELING OF NEUTRON STARS}

Finn (MN 232, 259; MN 227, 265) has investigated the occurrence of nonradial relativistic stellar pulsations. McDermott et al. (ApJ 325, 725) examine nonradial oscillations in neutron star models, including the occurrence of modes due to crustal elasticity, along with the usual $f_{-}, p^{-}$and $g^{-}$ modes. McDermott \& Taam (ApJ 318, 278) determine the nonradial g-modes in x-ray bursting neutron stars and find them to be sensitive to the envelope temperature. Lindblom \& Splinter (ApJ 345, 925) examine the dipole oscillations of fully relativistic neutron stars and provide equations for the solutions for the dipole $p$-modes to be solved numerically. 


\section{Flare Stars}

(R. E. Gershberg and N. I. Shakhovskaya)

During last 3 years Flare Stars (FS) were the main or one of the main objects under consideration at several meetings. The following proceedings should be noted:

- The Midnight Sun conference, held in Troms, Norway, July 1-8, 1987, 'Activity in cool star envelopes.' edited by Havnes et al. and referred to hereafter as HPSS

- Proceedings of the 5th workshop 'Cool stars, stellar systems and the Sun;' held in Boulder, USA, July 7-11, 1987, edited by Linsky \& Stencel and referred to as LS

- Proceedings of the IAU Coll. 104. 'Solar and stellar flares,' held in Stanford, USA, Aug. 15-19, 1988, edited by Haisch \& Rodono and referred to as HR. The volume containing poster papers from this colloquium is issued as a Special Publication of Catania Astrophysical Observatory and referred to as HRPP

- Proceedings of the IAU Symp. 137, 'Flare stars in star clusters, associations and solar vicinity.' held in Byurakan. USSR. Oct. 23-27, 1989, edited by Mirzoyan et al. and referrred to as MPT.

Several reviews on different aspects of FS should also be noted: on stellar activity and rotation by Rodono (Lecture Notes in Phys. 292, 39), on time scales and energy of flares by Gershberg (Mem. S. A. It. 60, 263), on X-ray emission from stellar flares by Paliavicini (AA Rev. 1. 177, 1989), and on energy release in stellar flares (IAU Symp. 142. 'Basic plasma processes on the Sun, edited by Krishan \& Priest). on the role of accelerated particles in stellar flares by Grinin (Mem. S. A. It., in press), on photospheric magnetic fields of cool stars by Saar (IAU Symp. 132. 'The impact of very high S/N spectroscopy on stellar physics,' edited by de Strobel \& Spite), on impulsive processes in late dwarf atmospheres by Katsova \& Livshits (AJ USSR 68, vol. 1), and on radio emission from FS by Bastian (Sol. Phys., in press).

In the solar vicinity a number of new FS were found or suspected to be of this type: Zhang Zhousheng et al. (IBVS 3050) registered 2 fiares on the G5 star HD 97766: Tsvetkov et al. (Astrofizika 29, 74 and HPSS p. 77) concluded that one of the most active FS in the NGC 7000 region. $\mathrm{B} 19=\mathrm{V} 1589 \mathrm{Cyg}$, is not a member of the aggregate but belongs to the solar neighbourhood: Hawley et al. (AA 220, 218) discovered flare activity on Gl 277A; Schuster (IBVS 3256) observed a rather strong flare on the high-proper-motion star G-64-34: Doyle et al. (AA Suppl.. in press) detected seven flares on Gl 375 and one flare on Gl 431; both stars have strong emission Balmer lines. Barden (ApJ 317, 333) detected a BY Dra-iike binary companion to the contact binary XY Leo. Heckert et al. (BAAS 20.674) concluded that photometric data is consistent with a hypothesis that HD 80715 is a BY Dra system.

Results of patrol observations of known FS were published by Herr \& Opie (IBVS 3069), Herr \& Caputo (IBVS 3243). Herr \& Charache (IBVS 3270), Melikian et al. (IBVS 3338), Mavridis \& Varvoglis (IBVS 3429), Ichimura \& Shimizu (in press).

Page (Mount Tamborine Obs. Publ. 3) published the 'Atlas of flare stars within the solar neighbourhood' containing about 80 finding charts for FS with other information.

New FS in stellar clusters were discovered by Kelemen (IBVS 3103 and MPT p. 67). Tsvetkova \& Tsvetkov (IBVS 3189). McNaught (IBVS 3395), Konstantinova-Antova \& Tsvetkov (IBVS 3190), Szecsenyi-Nagy (Publ. Astron. Dep. Eötvös Univ. 9. 1988), Parsamian \& Chavira (in press), Chavushian \& Brutian (MPT p. 63), Sun \& Tong (Vistas Astron. 31, 385).

Tsvetkov \& Tsvetkova (IBVS 3366) carried out a cross-identification of FS in the Pleiades. Mirzoyan et al. (Astrofizika 29, 44 and MPT p. 59) concluded that the UV Cet type stars originated in already disintegrated systems. They estimated that the fraction of field FS in regions of stellar clusters is less than $10 \%$, that the total number of field FS in Galaxy is more than $4 \times 10^{9}$ and that of non-flaring red dwarfs is about $2 \times 10^{10}$ (Astrofizika 29. 531). Mirzoyan et al. (Astrofizika 31. 259 and MPT p. 121) considered the fraction of FS among red dwarfs as a function of a stellar luminosity and age of a system. Mirzoyan \& Natsvlishvili (Astrofizika 27, 605), Mirzoyan et al. (Astrofizika 28, 540) and Parsamian \& Organian (MPT p. 109) discussed slow flares in clusters. Mirzoyan \& Hambarian (Astrofizika 28, 375) concluded that differences between FS near the Sun and in clusters are due to differences of their ages. Hambarian (Astrofizika 28,149) found that Trapezium systems consisting of FS are on the average larger than those consisting of T Tau stars. Mirzoyan \& Mnatsakanian (MPT p. 77) suggested the existence of two groups of FS in Pleiades with different proper motions. Kelemen (HPSS p. 73) carried out photometry of the Pleiades FS, Parsamian \& Oganian (Astrofizika 30, 220 
and Bult. Byurakan Obs. 65, in press) carried out spectral classification for 455 FS in clusters and found such stars located both above and under the main sequence: Mirzoyan et al. (MPT p. 95) did not find significant differences in spectra of the Pleiades FS located above and under the main sequence. Szecsenyi-Nagy (HRPP p. 143) found significant flare frequency variations for two of the most active FS in the Pleiades and concluded (Astrophys. Space Sci., in press) that such activity variations are common for younger and oider dwarfs; he considered energetic properties of FS (HPSS p. 69) and proposed a method for data filtering of non-homogeneous flares records (MPT p. 71). Natsvlishvili (in press) estimated the total number of FS in the Orion cluster to be about 2000. Mnatsakanian \& Mirzoyan (Astrofizika 29, 32 and MPT p. 113) obtained an exact analytical solution of the flare prediction problem and found that predictions are impossible for times exceeding the time of available observations by a factor of two.

Doyle et al. (AA 208, 208) registered 10 flares on FS Gl 234 AB. The strongest flare had an amplitude $3.8 \mathrm{mag}$ in the $U$ band, a rise time of $45 \mathrm{~s}$, and a decay time of $20 \mathrm{~min}$. At the imputsive phase during 20-40s the flare radiation had been registered in near IR bands. Tovmasyan \& Zalinyan (Astrofizika 28, 75) and Zhilyaev et al. (MPT p. 35) registered stellar flares shorter than $1 \mathrm{~s}$. Byrne et al. (AA. in press) estimated the $U$ band flaring energy release on FK Aqr over 8 years and did not find variations of the value. Neither BY Dra type variations nor transition region line flux variations were detected, but the MgII line flux decreased by about 25\%. Andrews \& Marang (AA, in press) registered 2 large flares on FL Aqr, determined its rotational period. suspected two active longitudes on the star. estimated the total area of starspots to be about $5 \%$ of its surface and suggested an activity cycle from 10 to 18 years. Doyle \& Mathioudakis (AA 227, 130) found that flares on YY Gem out of eclipse are much more powerful than that during eclipses, and Doyle et al. (AA, in press) suggested a periodicity in the flaring rate on this FS. Mavridis \& Avgoloupis (HPSS p. 65) found that during the EV Lac activity cycle its flare energy spectrum parameters vary significantly. Ishida et al. (AA, in press) and Ishida (MPT p. 43) studied flare activity of FS YZ CMi, AD Leo and EV Lac from 1971 to 1988 , defined mean colour indices of flare radiation $U-B=-0.98+0.17$ and $B-V=0.05+0.13$ for flare maxima of rather strong flares, and constucted flare energy spectra for these FS. Falchi et al. (Astrophys. Let., in press) registered flares on V1216 Sgr and V1054 Oph and concluded that differences between solar and stellar flares may be a consequence of biased observations since FS are monitored in continuum photometric bands while solar flares are detected in chromospheric line emissions.

Panov \& Korhonen (IBVS 3064) obtained a time evolution of colours of two flares on EV LaC and Panov et al. (AA Suppl, 75, 53) presented five-colour photometry of the EV Lac flares. Panov et al. (MPT p. 27) registered rapid spike flares on AD Leo and EV Lac, Zalinian (IBVS 3142) detected a flare on EV Lac with an unusual light curve. Pettersen et al. (MPT p. 15) did not find significant variations of the $A D$ Leo flare frequency from 1972 to 1988 . but suggested a possible cyclical behavior of the UV Cet flare activity with a period between 10 and 15 years (Proc. 6 Conf 'Cool stars. stellar systems and the Sun.' 1989, in press). Pettersen (MPT p. 49) concluded that the flare activity levels of dMe stars decrease as one approaches the lower end of the main sequence. In dM stars the upper limit for activity is about $1 / 1000$ of that in dMe stars. Cutispoto et al. (IBVS 3102) obtained the light curve of BY Dra in 1986 and constructed the two-spot model with round spots. Bondar (MPT p. 55) studied the plate collection of the Sternberg Institute from the end of the 19th century and suspected slow variations of mean brightnesses for 6 of the 13 FS examined.

Andrews (IBVS 3197; 3198; 3203; 3248; 3303; AA 210, 303; 214, 220; 227, 456; 229, 504 Armagh preprint $N$ 97) modified the auto-correlation technique and found quasi-periodicities ranging from a few minuntes to $6 \mathrm{~s}$ from the $U$ band observations of several FS. These quasi-periodicities are tentatively related to X-ray fluxes but not to $\mathrm{MgII} k$ line fluxes and might be connected with the lonson-Mullan MHD wave resonance regime of coronal loop heating. Chugainov \& Lovkaya (Bull. Crimean Astrophys. Obs. 80, 63) concluded that low amplitude periodical $\left(10^{3}-10^{4}\right.$ s) light variations observed in BY Dra may be due to the Shibahashi mechanism of the p-mode enhancement of global oscillations by magnetic fields.

Carter et al. (MN 231, 49) registered time-resolved spectra of a flare of an Orion cluster FS that resembled those of dMe stars.

Burnasheva et al. (HR p. 67 and AJ USSR 66. 328) registered the development of the CIV 1550 A emission in the EV Lac flare with a time resolution of about $1 \mathrm{~s}$ and detected a strong, fast burst. Houdebine et al. (AA, in press) discovered in the spectrum of the AD Leo flare a strong enhancement of the blue wing of the $\mathrm{H} \gamma$ profile and concluded a mass ejection event with a velocity up to $5800 \mathrm{~km} /$ $\mathrm{s}$ starting with the onset of the flare. Mathioudakis \& Doyle (AA, in press) registered strong increases of the CIV. HeII and continuum fluxes during UV monitoring the FS Wolf 630 and estimated $n_{e}$ and 
radiative losses of the flare. Hawley \& Pettersen (ApJ, in press) observed a giant flare on AD Leo that lasted more than $4 \mathrm{~h}$ and estimated the contribution for the flare radiation in the continuum and lines within the range of 1200 to $8000 \mathrm{~A}$ at the impulsive and gradual phases. From studies of several flares they concluded that flare emission features are produced under similar atmospheric conditions regardless of the total flare energy. The range in the total emitted energy may thus be primarily a function of a flare area and its duration but not of large differences in a flare heating rate and resulting atmospheric structure. Phillips et al. (MN 235, 573) found only modest enhancement in the IUE spectra during a rather strong flare on UV Cet when Balmer lines showed a broadening with red wings up to $100 \mathrm{~km} / \mathrm{s}$. Doyle \& Cameron (MN. in press) registered in the Gl 890 spectra a dip in MgII line flux and a shift of the $\mathrm{H} \alpha$ line bisector that may be interpreted as a pass of a neutral hydrogen cloud in front of the stellar disk. Fekel (AJ 95, 215) and Fekel et al. (AJ 96, 1426) studied chromospheric activities of the BY Dra-type binaries V478 Lyr and LR Hya. V478 Lyr is a G8 star, which is one of the earliest BY Dra-type variables. Vilhu et al. (AA 222, 179) studied activities of CM Dra and Rossiter 137B that are expected to be almost totally convective, and concluded that the data obtained fit a rotationdependent distributed dynamo generating magnetic flux in stars of such inner structures.

De Jager et al. (AA 211, 157) found a close similarity between the impulsive phase of the large flare on UV Cet and the model proposed for solar flares but with fundamental quantitative differences: the stellar flare zone responsible for optical emission had a vertical extent more than $700 \mathrm{~km}$ compared to $10-20 \mathrm{~km}$ on the Sun, its optical thickness exceeded unity at least for 6 min after the flare maximum while the solar value is less than $10^{-2}$. They estimated $n_{e}=10^{15} \mathrm{~cm}^{-3}$ and $T=16000 \mathrm{~K}$ for the optical flare at maximum and found a strong inhomogeneity of the hot component responsible for the X-ray flare with temperatures from 10 to $40 \mathrm{MK}$ and a magnetic field strength less than $500 \mathrm{G}$.

Butler et al. (HPSS p. 167) found almost continous variability of $\mathrm{H} \gamma$ and $\mathrm{U}$-band fluxes from UV Cet and YZ CMi and some correlation for UV Cet between the optical radiation and soft $X$-rays. Ambruster et al. (HRPP p. 27) analyzed simultaneous optical and X-ray observations of EV Lac and concluded that the independence of chromospheric and coronal activities may be due to the existence of magnetically isolated regions in stellar atmospheres. Butler et al. (AA 206. L1) found a linear correlation between Balmer and the total soft X-ray emission from stellar and flares that extends over 4 orders of magnitude.

Haisch et al. (AA 181, 96) found that during the EQ Peg flare the MgII $h$ and $k$ line flux changed only about $10 \%$, the flare area was about $1.5 \%$ of the stellar surface and the soft $X$-ray curve had a rise phase lasting longer than the decay. In the $Y Z$ CMi flare Doyle et al. $(A A 193,229)$ found an excess emission in wings of Balmer lines that may be due to matter moving with a velocity up to 300 $\mathrm{km} / \mathrm{s}$. They also found that the X-ray flux showed only a moderate dependence on Balmer lines and none on the U-band variations. Doyle et al. (AA 191.79) found that in the Wolf 630 flare the $\mathrm{H} \alpha$ flux was about $4 \%$ of the total $X$-ray flux and the flare had 2 to 3 loops with heights of about $10^{9} \mathrm{~cm}$ and $\mathrm{n}_{\mathrm{e}}$ near $10^{12} \mathrm{~cm}^{-3}$. Haisch et al. (AA, in press) confronted coronal and chromospheric flare energies and similar quiescent state energies for the FS Proxima Cen and found a flare and microflare related process heating the corona consistent with the concept of an X-ray heated chromosphere. Byrne \& McKay (AA 223, 241) re-analyzed the Proxima Cen flare and concluded that coronal X-rays and transition region UV line emission arose in comparable volumes and radiative losses due to this emission were also comparable.

Caillault and Zoonematkermani (MPT p. 159) examined 172 EINSTEIN $X$-ray sources in the Orion cluster but did not detect strong flares. Pallavicini et al. (AA 227, 483) detected flaring and quiescent $X$-rays from Castor and suggested that they originate from an unseen late-type companion. Tagliaferri et al. (AA, in press) registered an X-ray flare on van Biesbroeck 8 that was not known as a FS: its quiescent $X$-rays were also detected. This implies that stars later than M5 can be active X-ray emitters. Pollock et al. (AA, in press) concluded that both components of $\mathrm{Gl} 867 \mathrm{AB}$ radiate quiescent and flaring X-rays. Agrawal (AA 204, 235) found X-ray variations from Gl $867 \mathrm{~A}$ and represented the data by a two-temperature model. O'Dea \& McKinnon (PASP 99. 1039) determined upper limits to a quiescent $X$-ray flux from Wolf 359 . Rao \& Vahia (AA 188, 109) identified 11 objects with FS and RS CVn stars among fast transient $X$-ray sources registered with the Ariel $V$ satellite. Rao and Singh (ApJ 352,303 ) detected $X$-rays from the fast rotating FS Gl 890 and Rao et al. (ApJ, in press) observed flaring and quiescent $X$-ray emission from the BY Dra-type star $B D+48^{\circ} 1958 A$. In both cases the $X$ ray luminosities and plasma temperatures were typical of a dMe FS. Hudec et al. (Bull. Astron. Inst. Czech 39, 296) concluded that the X-ray source EXO $020528+1454.8$ belongs to dMe FS. Pizzichini et al. (HE-UHE behavior of accreting $X$-ray sources. p. 83,1987 ) suggested that the M-type high proper motion star G 227-022 is an optical counterpart of the X-ray source and a FS. 
Pallavicini et al. (AA 191, 109) concluded that a continuous temperature distribution does exist in coronae of late-type stars. Even during quiescent conditions it extends to more than 10 MK. Pallavicini et al. (AA 228, 403. HPSS and MPT p. 147) presented a survey of X-ray observations of FS carried out with the EXOSAT Observatory. Analysis of data, based on nearly 300 hours of monitoring more than two dozen sources, lead to conclusions that (i) the quiescent $X$-ray luminosity of FS is not dependent on stellar rotation but on bolometric Juminosity; (ii) there exists variability in the $X$-ray region in the form of flares as well as more gradual variations; (iii) there exists a large variety of time scales and energies for stellar $X$-ray flares and, possibly, two classes of events similar to solar compact and two-ribbon flares should be considered; (iv) no evidence in favour of $X$-ray microflaring activity is found.

Fleming et al. (ApJ 340. 1011) found the correlation $L_{x} \times(v \sin i) 1.05 \pm 0.08$ and concluded the existence of an upper limit to the coronal activity determined by the stellar radius. Caillault (PASP. in press) found that $M$ dwarfs contribute less than $10 \%$ to the soft $X$-ray background.

Kundu et al. (AA 195, 159) observed 4 FS with the VLA and EXOSAT. All stars were detected during the quiescent state and during flares. However, there was little correlation between two wavelength domains. Gary et al. (LS p. 106) found that optical emission of the AD Leo flares is correlated with CIV, while radio emission is better correlated with Hell. The $6 \mathrm{~cm}$ emission from one of these flares was initially $100 \%$ polarized (Byrne \& Gary. HRPP p. 63). Kundu et al. (ApJ 312. 822) observed UV Cet. AT Mic and AU Mic at 6 and $20 \mathrm{~cm}$ during flares and in quiescent states and proposed models for a quiescent microwave-emitting corona. Kundu \& Shevgaokar (ApJ 334, 1001) registered radio emission from $\mathrm{YZ} \mathrm{CMi}$ at 20 and $90 \mathrm{~cm}$ and concluded a coherent nature of the $90 \mathrm{~cm}$ radiation. Willson et al. (AA 199. 255) detected radio emission at $6 \mathrm{~cm}$ from one (Gl 735) of 16 nearby $M$ dwarfs studied. Willson (ApJ 326. 300) obtained frequency spectra of the $Y Z$ CMi radiation near $1465 \mathrm{MHz}$ and interpreted the data in terms of electron-cyclotron maser emission. Jackson et al. (LS p. 103) carried out a survey of FS at 6 and $20 \mathrm{~cm}$ and detected radio emission from 9 of the 27 objects studied. Spencer et al. (in preparation) detected the YZ CMi flare as well as continuum emission at $6 \mathrm{~cm}$. Microflaring with pulses shorter $20 \mathrm{~s}$ seems to occur at rates about 1 per hour.

Bastian et al. (ApJ 353, 265) recorded dynamic spectra of FS radio bursts and found that they display a rich structural variety, including spikes with rise times less than $20 \mathrm{~ms}$ and brightness temperatures of about $10^{16} \mathrm{~K}$. Quasi-periodic pulsations were also observed. In some cases sudden flux reductions were registered. It is suggested that cyclotron maser instability operating in an inhomogeneous medium is responsible for radio emission from FS. Güdel et al. (AA 220, L5) and Benz et al. (MPT p. 139) used 3 large radio telescopes and resolved the AD Leo flare emission into a multitude of broadband pulsations with rise and decay times of about $0.1 \mathrm{~s}$ or less and with circular polarization near $100 \%$. Güdel \& Benz (AA 211, L5) detected non-flaring radio emission of UV Cet from 1.3 to $20 \mathrm{~cm}$ and found a minimum or break at $3.6 \mathrm{~cm}$. Bastian et al. (AJ 95, 794) observed FS in the Pleiades and Caillault observed FS in the Hyades, but only upper limits of their microwave emission were obtained. Caillault (AJ 95,887 ) observed 7 very rapidly rotating BY Dra-type stars and didnot find a strong correlation between microwave emission and rotationally modulated optical brightness.

The quiescent state of FS has been activily studied as well. Doyle \& Butler (AA, in press) carried out UBVRIJHKL photometry of about $90 \mathrm{~K}$ and $\mathrm{M}$ dwarfs and estimated their bolometric luminosities, effective temperatures and radii. Tsikoudi (AJ 95. 1797) considered IRAS data and identified 15 FS at $12 \mu \mathrm{m}$ and half of them show a weak $25 \mu \mathrm{m}$ emission also. Mullan et al. (ApJ, in press) found that at 12 $\mu \mathrm{m}$ FS are about $70 \%$ brighter than dM stars of the same $\mathrm{K}$ magnitude and $27 \%$ of $\mathrm{FS}$ that are the sources at $12 \mu \mathrm{m}$ have been detected at $100 \mu \mathrm{m}$ as well. while comparable $\mathrm{dM}$ stars have not been detected. Caillault \& Patterson (AJ, in press) redetermined temperatures and radii of about 40 nearby $M$ dwarfs and obtained a mass-radius relation that agrees with the theoretical relation to better than $10 \%$.

Pettersen (AA 209, 279) found variations of FS line profiles on time scales of months and years. estimated $n_{e}$ and radiative powers of stellar chromospheres together with variations of these values along the spectral sequence, and discussed the dependence of the values on stellar rotation and ages. Pettersen \& Hawley (AA 217, 187) carried out a spectroscopic survey of FS in the optical wavelength range and measured strengths of chromospheric emission lines and photospheric molecular features. Sundland et al. (HPSS p. 61) determined radiative losses in chromospheric and transition-region emission lines for $A D$ Leo in its quiescent state. Ambruster et al. (AA 208. 198) measured the MgII $h$ and $k$ emission-line widths in the $A D$ Leo spectrum and supported a 5 magnitude linear extension of the MgII Wilson-Bappu relation from brighter stars to low-luminosity dwarfs. Spectral studies of chromospheric activity of low mass stars were carried out by Cram \& Giampapa (ApJ 323, 316), by Cutispoto \& Giampapa (PASP 100, 1452), Giampapa et al. (ApJ 345, 536). Fleming \& Giampapa (ApJ 346, 299), and Stauffer et al. (ApJ, in press). 
Gershberg et al. (MPT p. 19) found changes in the amplitude of periodic brightness variations and in the Hox emission-line intensity for EV Lac between 1986 and 1987. Byrne \& McKay (AA 227. 490) found evidence for rotational modulation of the Mgll line flux in the spectrum of the very rapidly rotating FS GI 890: the maximum of the MgII line flux is near the phase of maximum spottedness. Haisch et al. (AA, 230, 419) analyzed the EXOSAT and IUE data of YY Gem and improved the epoch and period of the system. They also found the stability of the Mgll flux over three years (possibly caused by the entire covering of the star by plages), while transition-region lines showed significant rotational modulation and the X-ray flux showed a fairly deep eclipse minimum.

In a series of papers the correlation between different emission features of stellar atmospheres were considered. Doyle (AA 218, 195) found a power-law correlation between the chromospheric $\mathrm{H} \alpha$ emission and coronal $X$-rays for a group of $\mathrm{dM}$ and dMe stars. Byrne \& Doyle (AA 208, 159) discussed the relative importance of $L_{\alpha x}$ and $\mathrm{Mgll}$ lines as chromospheric coolants for $\mathrm{dM}$ and dMe stars, concluded that the transition from $M$ to Me dwarfs is related to the increase of the fraction of the surface covered by solar-like active regions and showed that X-rays. HeII $1640 \mathrm{~A}$ and $\mathrm{CIV} 1550 \mathrm{~A}$ line fluxes are tightly related over 3 orders of magnitude. Doyle (AA 214, 258) estimated the total radiative losses between $10^{4}$ and $10^{8} \mathrm{~K}$ in several late-type stars. Mathiodakis \& Doyle (AA 224, 179) compared surface $\mathrm{X}$-ray and Mgll fluxes for a group of $\mathrm{dMe}, \mathrm{dKe}, \mathrm{dM}$ and $\mathrm{dK}$ stars and did not find flux correlations for the whole group (although a good correlation is present for a subgroup $\mathrm{dMe} / \mathrm{dKc}$ stars). Some results for the radius-dependent upper limits of the $\mathrm{MgIl}$ and $\mathrm{X}$-ray luminosities were discussed. Doyle et al. (AA 228 . 443) found that in dM stars $L x$ is of approximately the same magnitude as MglI, but in dMe stars $L \alpha$ is at least a factor of 2 more important and contributes about $25 \%$ of the total chromospheric losses. about the same as the $\mathrm{H}_{c}$ line. Byrne \& Doyle (AA, in press) found that in $\mathrm{H} \alpha$ absorption stars the fluxes of transition-region emission-line surface are weaker by more than an order of magnitude than in typical dMe stars and comparable to those of the quiet Sun.

Rutten (AA 177, 131) considered the relation between Call and Mgll chromospheric and X-ray coronal flux densities and rotational periods with the colour index. Schrijver \& Rutten (AA 177, 143) found that $M$ dwarfs show deficiencies in chromospheric fluxes as compared to earlier stars of the same rotation period but no deficiency in transition-region line fluxes.

Several theoretical studies on FS have also been carried out. Poletto (HR p. 313) applied a reconnection model originally developed for solar two-ribbon flares to the EQ Peg data and concluded that stellar observations fit the model. Kopp \& Poletto (Proc. 6 Conf. 'Cool stars, stellar systems and the Sun,' 1989, in press) developed a simple model for a stellar analogy of compact solar flares to describe average thermodynamical properties of a flare loop as a function of time during a flare decay. Grinin \& Sobolev (Astrofizika 28, 355; 31, 527) developed a model of an impulsive phase of an optical stellar flare excited by a high energy proton beam: if the initial energy of protons is several MeV, such a beam can heat upper layers of a FS to the state that they will radiate similarly to a stellar flare at a maximum phase. Van den Oord (AA 207, 101) considered a possible role of proton beams in stellar flares and found that such a mechanism of energy deposition to a stellar chromosphere may explain the weak correlation between optical and microwave flares. Reale et al. (LS p. 179) computed the evolution of temperature. density, pressure and velocity within the framework of the Palermo-Harvard hydrodynamic numerical code to reproduce the X-ray observations of the 1980 Aug. 20 flare on Proxima Cen. Hawley (Mem. S. A. It., in press) developed a set of chromospheric models featuring a coronal loop geometry, energy. balance throughout the entire loop from photosphere to corona, and rigorous treatment of the radiative transfer in the optically thick chromospheric emission lines. The models show that the soft $X$-ray and thermal conduction from a long-lived hot corona are effective heating agents in the lower atmosphere during the gradual phase of stellar flares, give the right order of magnitude for emission lines produced during the gradual phase of the flare with a reasonable coronal temperature evolution, and have implications for the white-light continuum radiation during impulsive phase of flares. Katsova \& Livshits (HRPP p. 71: AJ USSR 66, 307) analyzed high time resolution observations of the EV Lac flare and concluded that the fast CIV flux variations detected are the evidence of a shock wave formation and fast evaporation of the chromosphere.

Hayrapetyan et al. (Proc. Workshop 'Reconnection in space plasma.' ESA SP-285. 163; Astron. Circ. USSA 1529 \& 1537) suggested a new pinch model of a primary energy release in solar and stellar flares that might explain the high energetic effectiveness and temporal scales of magnetic energy transformation into thermal and kinetic energies. Grandpierre (HPSS p. 159; HRPP p. 365) concluded that existing magnetic flare theories are inconsistent with a number of basic observational facts but the convective flare theory seems to be consistent with them. Gurzadyan (ApJ 332, 183) presented the basic formulation of the fast electron hypothesis according to which optical flares on FS are caused by 
non-thermal interaction between photospheric infrared photons and high-energy electrons appearing during flares above stellar surfaces.

Pustil'nik (Pis'ma Astron. Zh. 14. 940) gave a theoretical explanation for the observed frequency energy power law relation for the UV Cet type star flares in the framework of ideas that energy of flares relate to turbulent motions of magnetized photospheric matter.

Bruevich et al. (AJ USSR 67, 115) proposed a new algorithm to calculate the emission Balmer decrement for optically thick nonmoving media. Using the algorithm Katsova (MPT p. 321: AJ USSR, in press) estimated $n_{e}$ in flares and quiescent states of FS, and Katsova et al. (HRPP p. 87: MN. in press) found that the transition from a slope decrement to a steep one near a flare maximum is due to significant decrease of an optical thickness in the L $\alpha$ line center. Houdebine et al. (HRPP p. 59) estimated electron temperatures of flare plasmas from emission line fluxes assuming homogeneous, stationary and optically thin radiating matter. Houdebine \& Butler (MPT p. 313) studied the response of a quiescent chromosphere to a large radiation field arising from a flare and showed that a major part of Balmer line fluxes may arise from such a chromosphere rather than from a heated flare plasma itself.

Katsova (HPSS p. 245) analyzed X-ray data from 42 active late type dwarfs, obtained densities at bases of coronae and found that these densities increase from $\mathrm{G}$ to MO-M3 stars and then decrease. This might be caused by different mechanisms of coronal heating.

\section{T Tauri Stars}

(Gibor Basri)

The understanding of T Tauri stars (TTS) which was just coming into focus in the last report has progressed and solidified substantially in the last 3 years. There is now a consensus view that low mass pre-main sequence stars manifest themselves as two basic observational types: the weak-lined TTS whose characteristics include weak $\mathrm{H} \alpha$ emission less or equal to $10 \mathrm{~A}$ equivalent width) and very weak or absent near-infrared excesses (WTTS), and the classical TTS. with stronger to very strong line emission and strong infrared and uitraviolet excesses (CTTS). There is in fact a continuum of cases between these two somewhat artificial categories, but most stars lie fairly clearly in one or the other. The evidence is increasingly strong that the fundamental difference between the two classes is the presence of accretion from a disk, and that most of the spectral characteristics particular to CTTS are directly or indirectly a result of this accretion. The classes share in common those characteristics which can be ascribed to the star by itself.

Because the field has been so active, a number of conferences and reviews have occured which collectively encompass most of the progress that has been made. The conferences of note are 'Formation and Evolution of Low Mass Stars' (NATO-ASI, eds. Dupree \& Lago, 1988); 'Low Mass Star Formation and Pre-Main Sequence Objects' (ESO Workshop, ed. Reipurth. 1989): 'Protostars and Planets III' (eds. Matthews \& Levy, Univ. Arizona Press, 1990); and 'Physics of Star Formation and Early Stellar Evolution' (NATO-ASI in prep.. 1990). Additionally, two major reviews have appeared by Bertout (Ann. Rev. Astron. Astrophys. 27, 351, 1989) and Appenzeller \& Mundt (Astron. Astrophys. Rev. 1, 291. 1989). These do not comprise the complete list of substantial new collections on T Tauri stars, but are among the major ones. Similarly, in the details below I do not cite all references covering a particular topic; the selection is somewhat personal and does not imply that uncited papers are any less significant (see the sources above for more complete citation lists). Finally, this review concentrates on results from optical and near infrared observations: the related topics of Herbig-Haro objects and optical jets, and much interesting theoretical progress and longer wavelength observations of the surrounding molecular clouds have been omitted.

\section{THE STARS THEMSELVES}

The last three years have seen the publication of the majority of the work on WTTS. Walter et al. (AJ 96, 297. 1988) published the main collection of them for Taurus and showed they are similar to the stellar components of CTTS. These stars have largely been found as serendipitous X-ray sources. Feigelson \& Kriss (ApJ 338, 262, 1989) give a smaller collection for the Chameleon cloud and Strom et al. (ApJ, in press) give results for the Lynds 1641 cloud. These all show that the WTTS are fairly well mixed with the CTTS both spatially and in the HR diagram, although there is a subset of older and/or more spatially dispersed WTTS. The latter paper re-examines the question of whether CTTS show a 'quenching' of $X$-rays due to strong mass outflow, and concludes they do not. Bouvier (AJ 99, 946 , 1990 ) shows that the $X$-ray activity obeys the same sort of relation to stellar rotation as in the RS CVn stars, making it likely to be due to stellar coronae. 
A major paper continuing the detection of starspots on both CTTS and WTTS was published by Bouvier \& Bertout (AA 211, 99, 1989). They show that the spot phenomenon is fairly widespread among TTS; though easier to find on WTTS which do not suffer from the confusion of light changes due to unsteady accretion. The spots seem to have the same general characteristics as those on RS CVn stars; coupled with the $X$-ray results above and previous studies of $T$ Tauri chromospheres it now seems fairly clear that TTS are very magnetically active. but probably are not qualitatively different from the most active of older stars. The work on rotation of TTS makes this reasonable; they are slowly rotating compared to breakup, but rapidly rotating compared to older stars in that part of the HR diagram (except short-period RS CVn stars). On the other hand, the case of RY Lup (Gahm et al. 1989. AA 211. 115) keeps us aware that periodic light variations do not always imply starspots.

One of the major areas of progress in the last three years has been in the study of binaries among the TTS. Until the last few years, there was a disturbing lack of known binaries. given the fact that most older stars are in binary systems. This has now largely been eliminated through modern observations. These results are summarized by Zinnecker in the ESO Workshop mentioned above. The new techniques include CCD and NIR imaging. NIR speckle interferometry and lunar occultation (Simon et al. 1987, ApJ 320. 344: Chen et al. 1990. ApJ 357. 224). These results seem to be bringing the incidence of wide binaries into accord with expectations. The situation regarding spectroscopic binaries also has received a boon with the discovery of the WTTS. A number have now been found, and Mathieu et al. (AJ 98, 987, 1989) argue that their frequency is now not inconsistent with expectations, though more data is desirable. There may still be a problem with the CTTS (Mathieu et al. in prep), but the observational selection effects against their detection are most severe for these stars.

Finally, the advent of infrared imaging has greatly increased our power to find TTS hiding deeper behind the dust clouds of their parental molecular clouds. A number of major surveys have revealed many new sources. These include the $\rho$ Oph region (Wilking et al. 1989. ApJ 340. 823), Lynds 1641 (Strom et al. 1989. ApJ Suppl. 71, 183). Orion (Lada, PhD thesis; Johnson et al. 1990. AJ 100, 518), Taurus (Kenyon et al. 1990, AJ 99, 869) and others (e.g. Persi et al. 1990. AJ 99, 303).

\section{CIRCUMSTELLAR DISKS}

At the time of the last review, the disk paradigm for TTS was just catching on. Since then it has become the consensus explanation for CTTS, because of its power to explain observations across the electromagnetic spectrum in a simple and self-consistent way. A number of investigations have advanced it for the FU Ori stars (Hartmann \& Kenyon 1987, ApJ 312, 243), extreme TTS (Kenyon \& Hartmann 1987. ApJ 323, 714) and CTTS (Bertout et al. 1988. ApJ 330, 350). The simplest models have been extended to explain the Balmer continuum emission jumps often observed by making the boundary layer optically thin by Basri \& Bertout (ApJ 341,340, 1989), who also discussed the uniqueness of the models. The accretion rates observed range from a few $10^{-9}$ to a few $10^{-7}$ solar masses per year.

Substantial progress has also been made on the optical veiling of photospheric lines by the UV excess continuum; Hartmann \& Kenyon (ApJ 349, 190, 1990) published a collection of veilings from a narrow spectral range showing that accretion is a common characteristic of CTTS and assessing possible effects of disk accretion on early stellar evolution. Kenyon \& Hartmann (ApJ 349. 197. 1990) also assessed its effects on observational determination of stellar parameters. Hartigan et al. (ApJ Suppl. 70. 899. 1989) made a detailed assessment of the veiling in BP Tau over a wide spectral range, and Basri \& Batalha (ApJ, in press) made extensive such measurements for a large sample of TTS. The correlation of $\mathrm{H} \alpha$ emission with optical veiling is good at low and intermediate strengths, but not so good at high strengths. Veiling measurements provide a reddening-free measure of the excess light in the optical, and seem to be quite consistent with the disk hypothesis (showing, for example, a good correlation with near infrared excesses). They will also serve as an extra constraint on disk parameters. Bouvier \& Bertout (in the ESO Workshop) made the first attempt to constrain disk parameters with a multi-dimensional chi-squared approach which demonstrates the extent to which they can be constrained. The simple disk accretion model has its greatest failure in explaining why the slopes of the infrared continua are as shallow (or even flat) as often observed. The discovery of possible $m=1$ gravitational instability modes by Adams et al. (ApJ 347, 959, 1989) provides a physically simple and consistent avenue for more massive disks to attain the shallower temperature gradients needed. Sub-millimeter surveys by Beckwith et al. (AJ 99, 924, 1990) and Adams et al. (ApJ 357. 606. 1990) show that flatter gradients are common. Most importantly, they provide fairly direct mass determinations for the disks which lie between a few hundredths to a few tenths of a solar mass; 
well above the minimum necessary to provide the mass of our own solar system. Strom et al. (AJ 97 , 1451, 1989) have made explicit the result that these disks do not last much beyond 10 Myr. which constrains the time in which putative solar systems must produce gas giant planets. Thus, the CTTS are now thought to provide current examples of the conditions needed to make planets, and have already begun to supply useful information on possible planet-formation mechanisms. The possible presence of inner holes in a few WTTS disks (Skrutskie et al. 1990. AJ 99, 1187) may even allow us to study the clearing of disks as it occurs.

\section{STRONG OUTFLOWS}

A great deal of new data on the strong emission lines in TTS has been gathered in the past 3 years. Much of it has not yet been published, but substantial work on the forbidden line emission has appeared. The survey by Edwards et al. confirms previous suggestions that red-shifted emission is rarely seen, and interprets that as the presence of the optically thick disks mentioned above. Along with the sub-mm work, this implies disk dimensions similar to our solar system. Strom et al. (AJ 95 . 534, 1988), Cohen et al. (ApJ 339, 455, 1989), and Cabrit et al. (ApJ 384, 687, 1990) demonstrate that the presence of the forbidden line winds is well-correlated with $H \alpha$, which in turn is thought to be well-correlated with disk accretion. The mass loss rates are not very well determined. but appear to be a substantial fraction of the mass accretion rates in individual systems. This subject is reviewed by Edwards et al. in 'Protostars and Protoplanets III'; for a discussion of wind mechanisms see Bertout et al. ('The Sun in Time', Univ. of Arizona Press, 1990).

In order to improve the diagnostics of the wind. the formation of the strong optical and NIR emission line profiles should be better understood. A major effort to evaluate the Alfven wind model in spherical geometry was carried out by Hartmann et al. (ApJ 349, 168, 1990). Similar mass loss rates were obtained, although the detailed match with the profiles was not very close. This group is working on studying the profiles in conical geometry, and reports better success. Natta (ESO Workshop and recent preprints) has pursued with collaborators the use of the NaD lines and NIR hydrogen lines, and also obtained similar mass loss rates. A large number of profiles gathered simultaneously with echelle spectrographs are currently in papers in preparation and these will be very helpful in constraining wind models near the star. They also show that the profiles can change on timescales of a day, sometimes dramatically. There is a convergence of observational and theoretical material suggesting that the wind mechanism is intimately tied to the interaction between the stellar magnetic field and the incoming disk. The interaction region is probably much more turbulent, complex, and extended (in strong emission lines) than a classical boundary layer, so the study of $\mathrm{T}$ Tauri boundary layers and the wind mechanism is really just beginning. 